\title{
TGF- $\beta$ and VEGF cooperatively control the immunotolerant tumor environment and the efficacy of cancer immunotherapies
}

\author{
Tristan Courau, ${ }^{1,2}$ Djamel Nehar-Belaid, ${ }^{1,2}$ Laura Florez, ${ }^{1,2}$ Béatrice Levacher, ${ }^{1,2}$ \\ Thomas Vazquez, ${ }^{1,2}$ Faustine Brimaud, 1,2,3 Bertrand Bellier, 1,2 and David Klatzmann 1,2,3 \\ ${ }^{1}$ Sorbonne Universités, UPMC University of Paris, Paris, France. ${ }^{2}$ INSERM UMR_S 959, Paris, France. ${ }^{3}$ AP-HP, Groupe \\ Hospitalier Pitié-Salpêtrière, Department of Biotherapies, Clinical Investigation Center in Biotherapy and Inflamation- \\ Immunopathology-Biotherapy Department (DHU 12B), F-75013, Paris, France.
}

\begin{abstract}
Tregs imprint an early immunotolerant tumor environment that prevents effective antitumor immune responses. Using transcriptomics of tumor tissues, we identified early upregulation of VEGF and TGF- $\beta$ pathways compatible with tolerance imprinting. Silencing of VEGF or TGF- $\beta$ in tumor cells induced early and pleiotropic modulation of immune-related transcriptome signatures in tumor tissues. These were surprisingly similar for both silenced tumors and related to common downstream effects on Tregs. Silencing of VEGF or TGF- $\beta$ resulted in dramatically delayed tumor growth, associated with decreased Tregs and myeloid-derived suppressor cells and increased effector $T$ cell activation in tumor infiltrates. Strikingly, co-silencing of TGF- $\beta$ and VEGF led to a substantial spontaneous tumor eradication rate and the combination of their respective inhibitory drugs was synergistic. VEGF and/or TGF- $\beta$ silencing also restored tumor sensitivity to tumorspecific cell therapies and markedly improved the efficacy of anti-PD-1/anti-CTLA-4 treatment. Thus, TGF- $\beta$ and VEGF cooperatively control the tolerant environment of tumors and are targets for improved cancer immunotherapies.
\end{abstract}

Conflict of interest: The authors have declared that no conflict of interest exists.

Submitted: December 11, 2015 Accepted: May 5, 2016

Published: June 9, 2016

Reference information: JCI Insight. 2016;1(9)::85974. doi:10.1172/ji.insight.85974.

\section{Introduction}

Effectors as well as regulatory antitumor immune responses are critical factors dictating tumor fate (1-3). In mice, the elimination of Tregs before tumor inoculation can lead to complete tumor eradication $(4,5)$. This indicates that, before tumor emergence, there are enough effector $\mathrm{T}$ cells (Teffs) to eradicate growing tumors, which they fail to do because of Treg suppression. In humans, composition of $\mathrm{T}$ cell tumor infiltrates is often a good predictor of tumor outcome (6), with a higher infiltration by Tregs mostly associated with poor survival $(7,8)$. Furthermore, recent successes of tumor immunotherapies using checkpoint inhibitors have established the antitumor potential of patients' Teffs (9-11), but also highlighted that without a boost these Teffs are inefficient in the tolerogenic tumoral environment $(12,13)$.

This environment is in large part imprinted by Tregs (14-16). At the time of tumor emergence in mice, competition arises between antitumor Tregs and Teffs (17). Importantly, the responding Tregs are specific for self-antigens and have a preexisting activated/memory phenotype (amTregs). This memory phenotype makes them respond more quickly than naive Teffs targeting tumor-specific antigens. In less than 4 days after tumor inoculation, amTregs imprint a powerful, dominant, tolerogenic environment. Adoptively transferred tumor-specific amTeffs block the development of tumors when injected between days 0 and 3 after tumor cell implantation, but lose this potential thereafter (17). Identifying the molecular drivers for this dominant tolerance would be of major importance, as it could provide new targets for (combined) cancer immunotherapies.

As tumor cells express a broad range of molecules that can impact Treg behavior (18, 19), we first examined the whole transcriptome of growing tumors at various time points after tumor implantation to identify genes and pathways modulated during tolerance imprinting. We identified VEGF and TGF- $\beta$ as good candidates, as their related pathways have an early modulation and they are pleiotropic cytokines with immunosuppressive activities, including effects on Tregs (20, 21).

VEGF is a major regulator of tumor angiogenesis (22), but also a powerful inhibitor of DC maturation 
Table 1. Highly significant reversal of the early downregulation of immune-related signatures by VEGF or TGF- $\beta$ silencing in B16 tumors

\begin{tabular}{|c|c|c|c|c|}
\hline Tumor & Signatures & All signatures & Immune-related signatures & $\begin{array}{c}P \text { values of enrichment } \\
\text { score }\end{array}$ \\
\hline \multirow[t]{3}{*}{ B16 } & Total & 4,635 & 233 & - \\
\hline & Upregulated & 345 & 0 & 1 \\
\hline & Downregulated & 1,330 & 99 & $4.5 e-06$ \\
\hline \multirow[t]{3}{*}{ B16-shVEGF } & Total & 4,615 & 233 & - \\
\hline & Upregulated & 322 & 54 & $2.2 e-16$ \\
\hline & Downregulated & 833 & 2 & 1 \\
\hline \multirow[t]{3}{*}{ B16-shTGF- $\beta$} & Total & 4,631 & 230 & - \\
\hline & Upregulated & 439 & 96 & $2.2 \mathrm{e}-16$ \\
\hline & Downregulated & 206 & 0 & 1 \\
\hline
\end{tabular}

The proportion of immune-related signatures among the significantly up- and downregulated ones was calculated in B16, B16-shVEGF, and B16-shTGF- $\beta$ tumor settings, as compared with control samples (tumor-free mouse skin), 4 days after tumor inoculation. The proportion test was performed regarding the total number of signatures tested using gene set enrichment analysis. The immune-annotated signatures were automatically counted using the grep function from $\mathrm{R}$.

that allows the accumulation of myeloid-derived suppressor cells (MDSCs) in the tumor (23, 24). VEGF can also induce Treg proliferation $(25,26)$ and inhibits the activity of Teffs in the tumor (27). By modifying tumor vascularization, VEGF could also alter the metabolic competition that affects the Treg/Teff balance and is a driver of cancer progression (28-30). TGF- $\beta$ is a master regulator of cellular proliferation (31). TGF- $\beta$ is also crucial for peripheral Treg induction from naive $\mathrm{CD}^{+}$cells $(32,33)$ and a major signaling molecule for the maintenance of Treg function (34), including in the tumor environment (35). It can also inhibit the activation and differentiation of both innate and adaptive immune cells, including NKs, DCs, and $\mathrm{T}$ cells $(36,37)$.

To study the role of tumor-derived VEGF and TGF- $\beta$ in antitumor immune responses and dominant tolerance establishment, we silenced their expression in tumor cells using lentiviral transduction of specific shRNA (38). We used poorly immunogenic B16F10 melanoma cells as well as more immunogenic AB1HA cells. Treg depletion in these two models has markedly different outcomes, with mostly a growth delay for $\mathrm{B} 16$ cells and an $80 \%$ eradication rate for AB1-HA cells (39).

We show that silencing of VEGF or TGF- $\beta$ overturns the tolerant environment of tumors. It unleashes natural antitumor immune responses that induce eradication of immunogenic tumors. It also confers on tumors a much higher susceptibility to both cellular- and checkpoint inhibitor-based immunotherapies. Thus, our study identifies TGF- $\beta$ and VEGF as the major drivers of tumor tolerance and targets for combined immunotherapies of cancer.

\section{Results}

Silencing of tumor-derived VEGF or TGF- $\beta$ induced early and pleiotropic upmodulation of immune responses detected in tumor tissue transcriptomes. Initial evaluation of WT tumor transcriptomics by microarray analysis suggested an early modulation of both VEGF- and TGF- $\beta$-related pathways (Supplemental Figure 1 ; supplemental material available online with this article; doi:10.1172/jci.insight.85974DS1). To evaluate their contribution to the dominant tolerance of tumors, we generated tumor clones silenced for each of these molecules by lentiviral-mediated transduction of specific shRNAs in B16F10 melanoma cells. We selected two stable clones (B16-shVEGF and B16-shTGF- $\beta$ ) having a greater than $95 \%$ reduction of expression of $V E G F$ or TGF- $\beta$ mRNA, respectively, and an in vitro doubling time similar to that of their parental cell line (Supplemental Figure 2).

To evaluate the early and global consequences of VEGF or TGF- $\beta$ silencing, we first analyzed the transcriptome of whole tumor tissues by microarray 4 days after tumor inoculation in WT mice (Table 1, Figures 1 and 2). Using gene set enrichment analysis (GSEA), we first performed an unsupervised enrichment analysis of compiled gene sets - lists of genes described as contributing to a given biological process - from the Gene Ontology database (40). Already at day 4, there were 1,675, 1,155, and 645 signatures (lists of genes whose expression variations are correlated) that were significantly modulated (FDR $P$ value $<0.05)$ in 
A

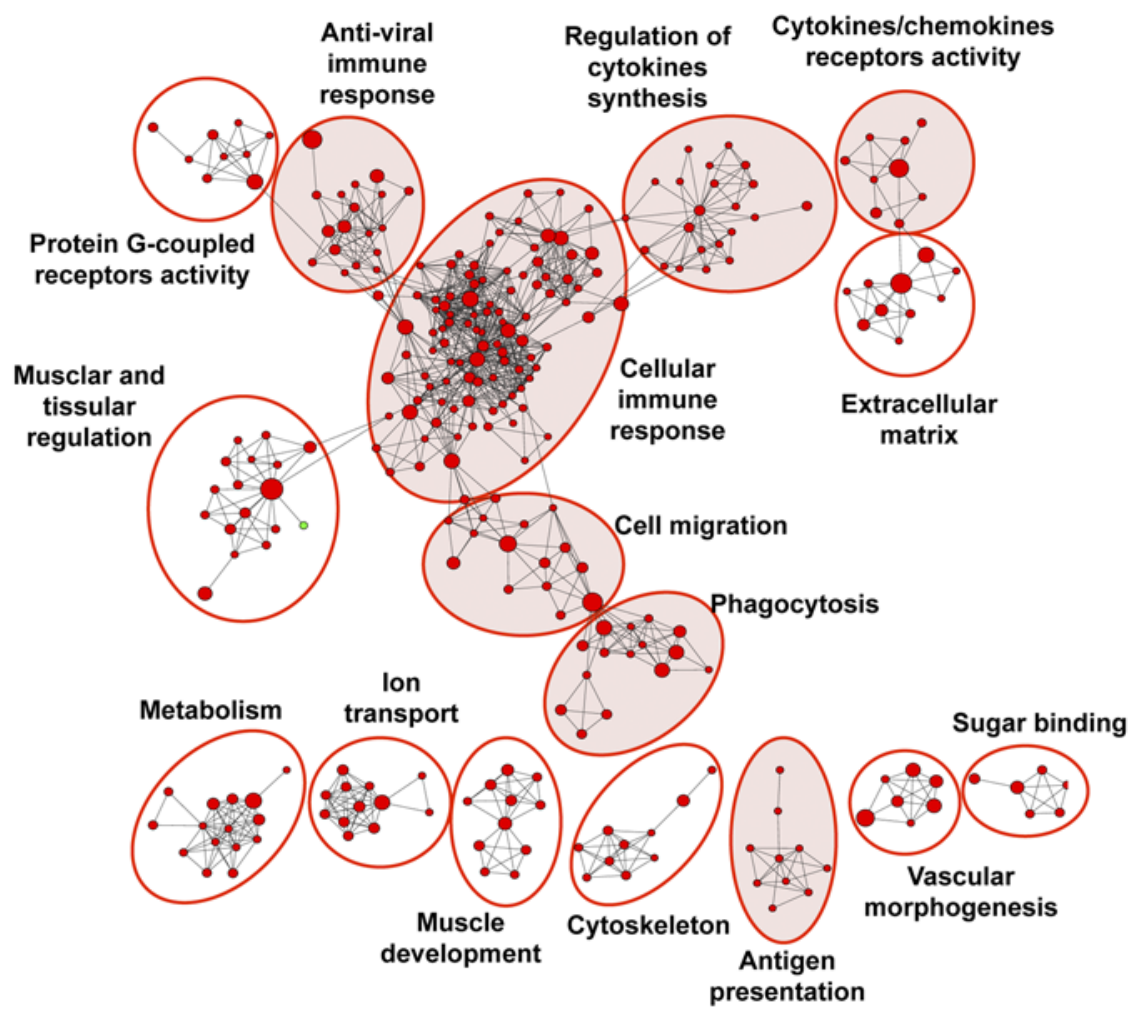

B

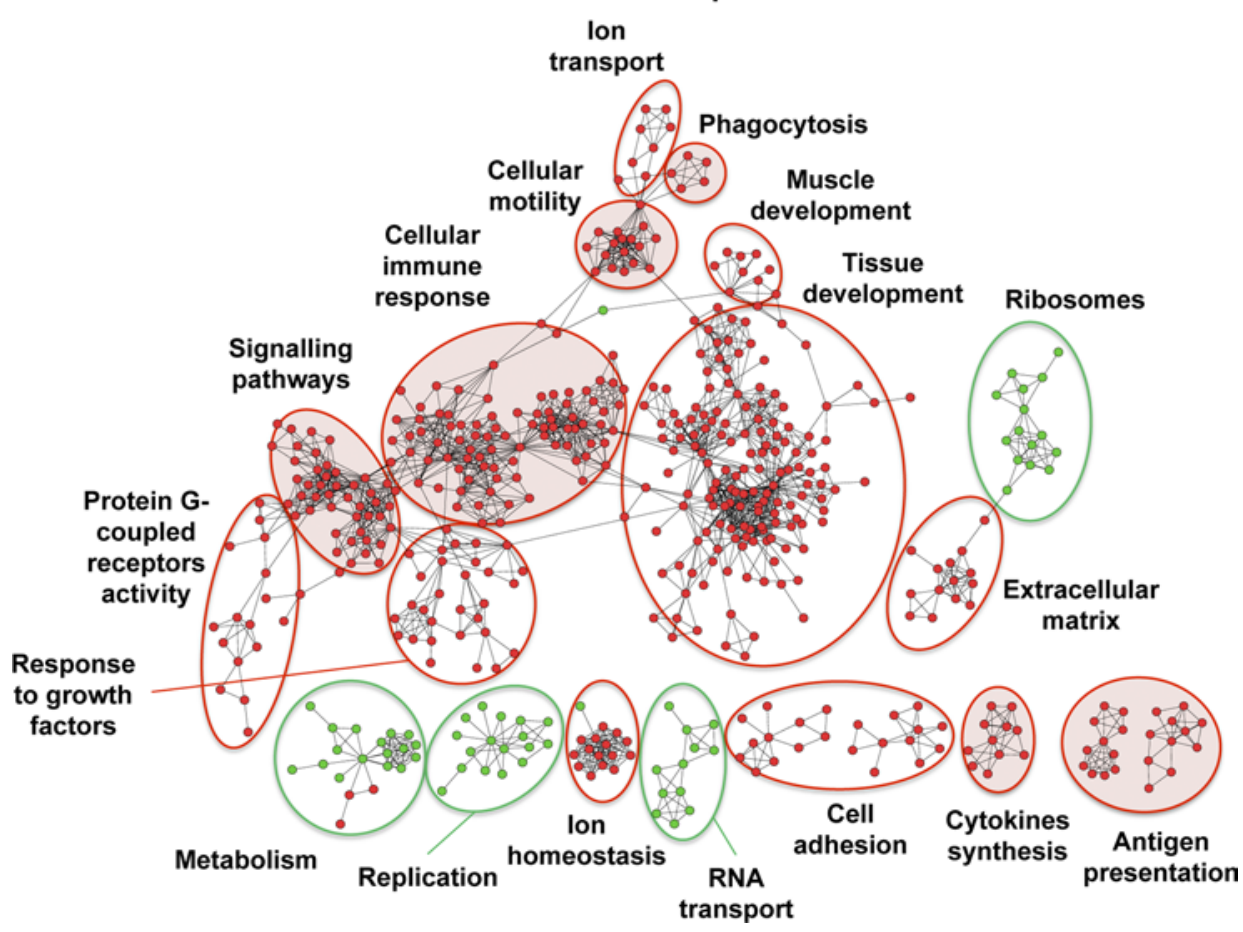

Figure 1. VEGF or TGF- $\beta$ silencing in B16 tumors reverses the early and pleiotropic inhibition of immune signature networks at the tumor sites. Microarray analyses of WT or silenced B16 tumor tissues obtained at day 4 after inoculation in C57BL/ 6 WT mice ( $n=6$ per group). Enrichments of Gene Ontology-compiled signatures were tested using gene set enrichment analysis and represented using Cytoscape software. (A and B) Cytoscape representations of significantly enriched signatures in (A) B16-shVEGF or (B) B16-shTGF- $\beta$ tumors as compared with B16 tumors. Each signature is represented by a node of size proportional to the number of genes composing it and color indicates down- (green) or upregulation (red). Networks of related signatures are edged and annotated for their main functions (modules). The immune-related networks are shaded in red.

B16, B16-shVEGF, and B16-shTGF- $\beta$ tumors, respectively (Table 1). Among these, 99 of 99 immune-related signatures of WT tumors were all downmodulated; in contrast, 54 of 56 and 96 of 96 immune-related signatures of B16-shVEGF and B16-shTGF- $\beta$ tumors, respectively, were highly significantly upregulated (Table 1). Using the Cytoscape software $(41,42)$, we then represented the significantly modulated signatures as networks (Figure 1). Compared with parental B16 tumors, VEGF or TGF- $\beta$ silencing induced marked changes in expression levels of only two categories of genes: those related to cell division (including metabolism) and those related to immune responses (Figure 1, A and B). The latter were all upregulated compared with B16 tumors, and we further evaluated them with ingenuity pathway analysis (Figure 2 ) and found that they could be linked to specific immune-related functions or settings. For example, most genes related to "lymphocytes migration" and "activation of T lymphocytes" are upregulated in both B16-shTGF- $\beta$ and B16-shVEGF tumors but downregulated in B16 (Figure 2, A-F).

To address whether these changes were linked to an early alteration of Treg responses, we analyzed our data sets with three Treg-related signatures described in the literature. Principle component analysis (PCA) showed that all of these signatures can differentiate the parental and the silenced tumors (Supplemental Figure 3).

In order to further validate the relevance of these findings, we attempted to test them on human data sets (Supplemental Figure 4). We generated molecular signatures using an unsupervised algorithm based 


\section{Lymphocyte migration}

A

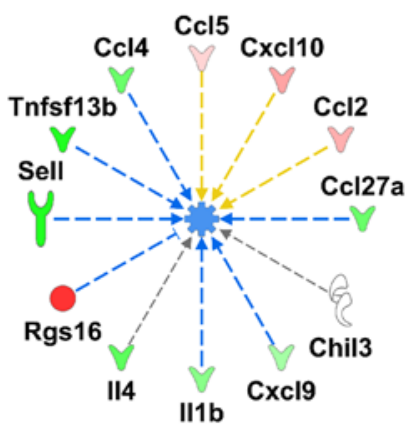

B

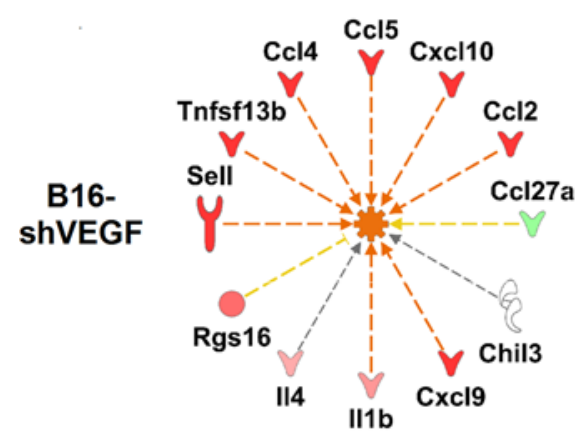

C

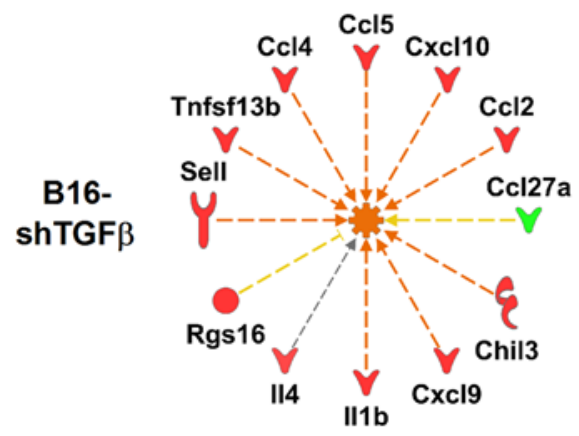

Activation of T lymphocytes

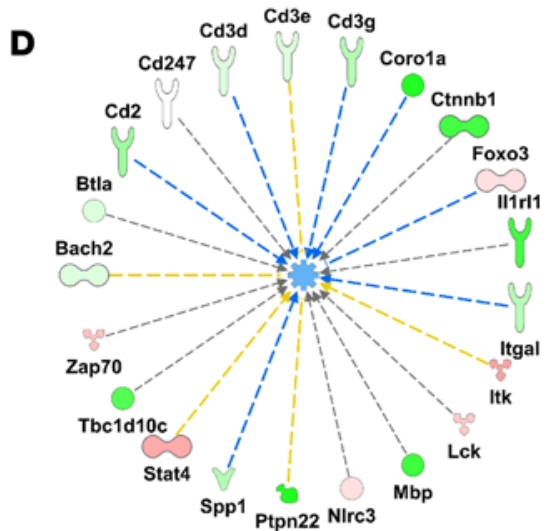

E

$\mathbf{F}$
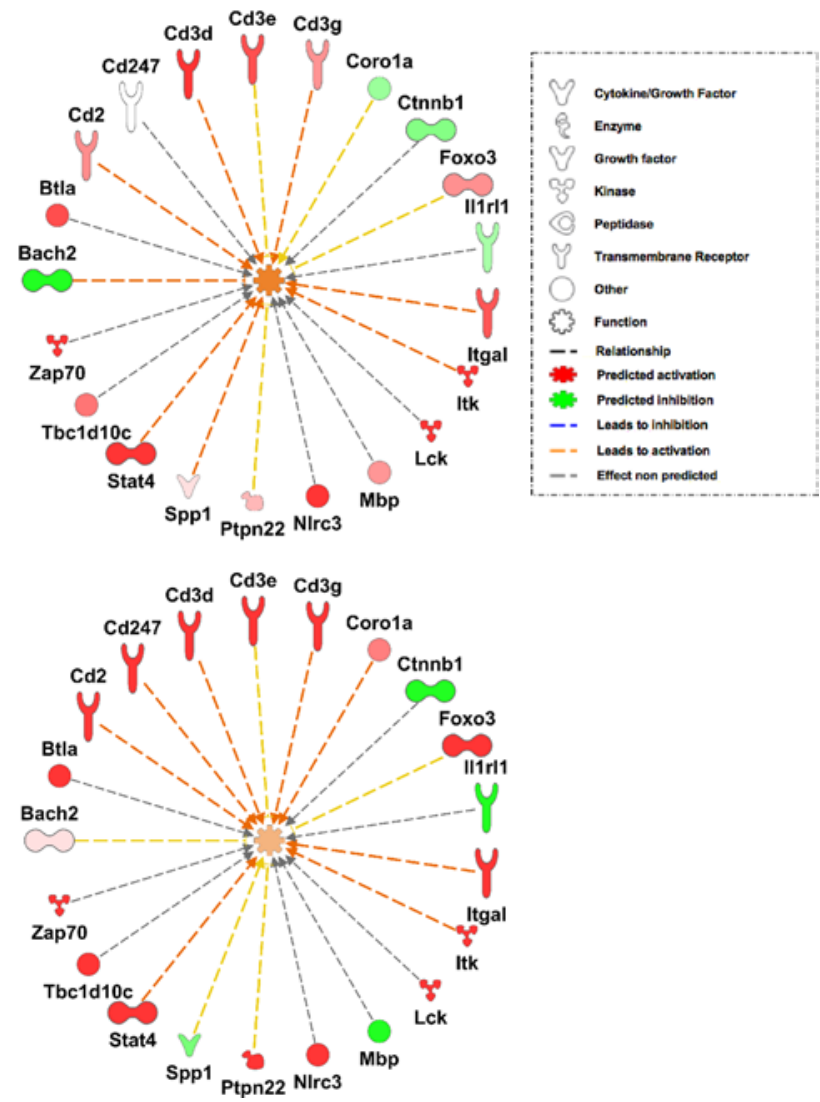

Figure 2. VEGF or TGF- $\beta$ silencing in B16 tumors reverses the early and pleiotropic inhibition of immune pathways in tumor sites. Microarray analyses of WT or silenced B16 tumor tissues obtained at day 4 after inoculation in C57BL/6 WT mice ( $n=6$ per group). (A-F) Circular representations of immunological functions from ingenuity pathway analysis (IPA): (A-C) "Lymphocyte migration" and (D-F) "activation of T lymphocytes". A given function is represented in blue if predicted to be downregulated and in orange if predicted to be upregulated by IPA software. Genes involved in these functions are plotted around them. Upregulated genes are shown in red and downregulated genes in green. Color intensity represents statistical significance.

on independent component analysis (ICA, ref. 43) and validated their significance using GSEA. We then selected the top three significantly modulated murine ICA-extracted signatures, converted them to their human orthologs, and compared them with a publically available human melanoma data set (Supplemental Figure 4A). In addition, we also tested signatures derived from immune-related modules presented in Figure 1 (Supplemental Figure 4B). Despite the much greater variability inherent to human studies, PCA showed very good separation between the 52 metastasis biopsies and the 31 primary melanoma biopsies, thus demonstrating the clinical relevance of our transcriptomic analyses.

Silencing of tumor-derived VEGF or TGF- $\beta$ unleashes T cell-mediated antitumor immune responses. To assess 
A

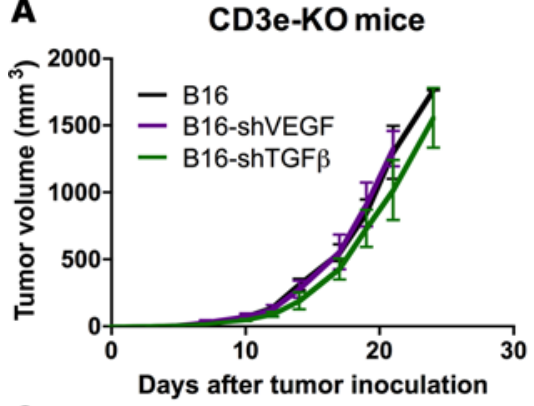

C

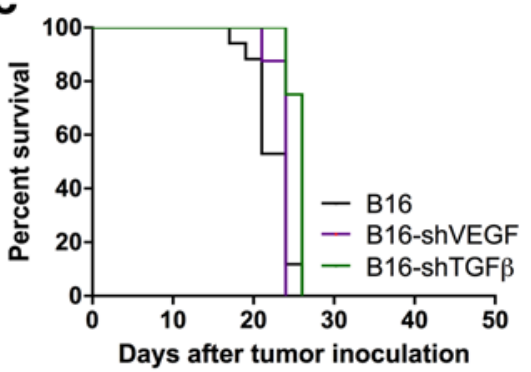

E

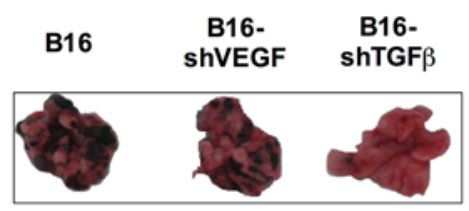

G

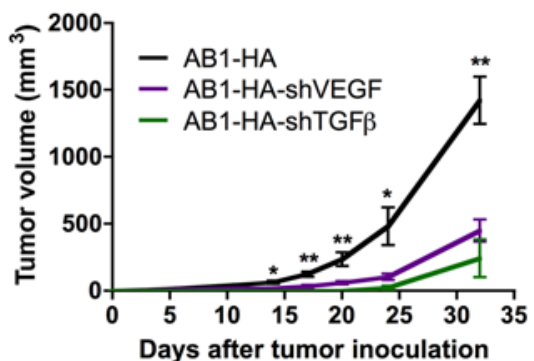

B

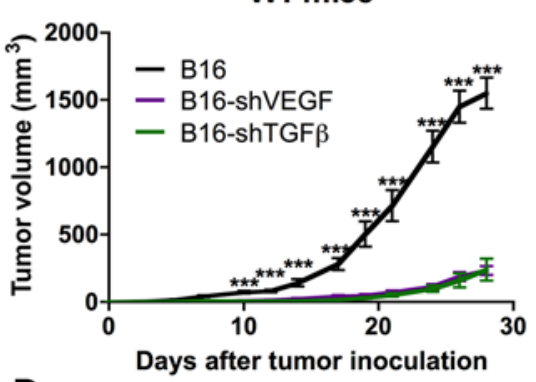

D

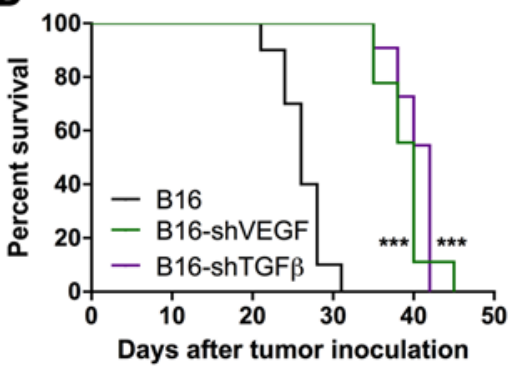

$\mathbf{F}$

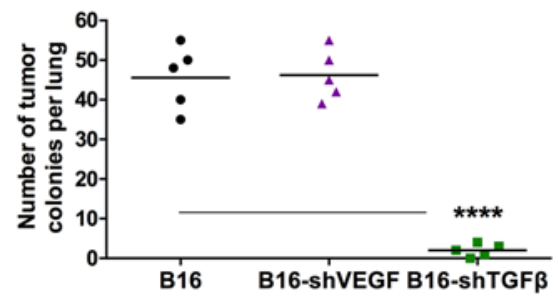

H

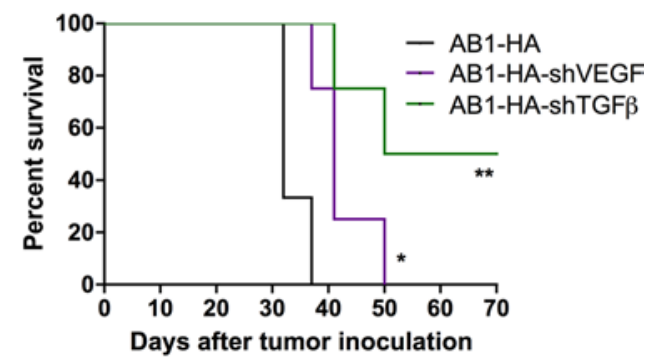

Figure 3. VEGF or TGF- $\beta$ silencing induces a T cell-dependent growth delay of B16 and AB1-HA tumors. ( $A$ and $B$ ) Kinetics and (C and $\mathbf{D})$ Kaplan-Meier survival curves of parental and silenced B16 tumor cell growth in (A and C) CD3 $\varepsilon-K O$ or (B and D) C57BL/6 WT mice after s.c. inoculation; $n=12$ mice per group in 3 independent experiments. (E) Representative lung images and $(\mathbf{F})$ quantification of tumor burden in the lungs of C57BL/ 6 WT 20 days after i.v. tumor inoculation of VEGF- or TCF- $\beta$-silenced or WT B16 tumor cells, $n=5$ per group. (G) Kinetics and (H) Kaplan-Meier survival curves of parental and silenced AB1-HA tumor cell growth in BALB/c mice after s.c. inoculation; $n=4$ mice per group. Statistical significance of the survival curves was analyzed by using the log-rank test, and the other results by using the Ordinary one-way ANOVA test with Bonferroni's correction ${ }^{*} P<0.05$; ${ }^{* *} P$ $\left.<0.005,{ }^{* * *} P<0.001,{ }^{* * * *} P<0.0001\right)$.

the relevance of the upregulation of immune-related signatures in silenced tumors, we next assessed their development in C57BL/6 immunodeficient and immunocompetent mice. There were no differences in the growth of B16-shVEGF and B16-shTGF- $\beta$ clones injected s.c. in $\mathrm{CD} 3 \varepsilon-\mathrm{KO} \mathrm{T}$ cell-immunodeficient mice (Figure 3, A and C). In contrast, there was a marked and comparable growth inhibition of both B16-shVEGF and B16-shTGF- $\beta$ tumors in immunocompetent mice (Figure 3, B and D). Tumor cells were also injected i.v., revealing that only TGF- $\beta$ silencing induced a dramatic decrease in their capacity to colonize lungs (Figure 3, E and F). In order to extend these results,

we also silenced TGF- $\beta$ and VEGF in AB1-HA tumor cells that are more immunogenic and from a different genetic background (BALB/c mice). We observed that VEGF and TGF- $\beta$ silencing led to a growth delay similar to that noted for B16 tumor cells (Figure $3 \mathrm{G}$ ), with a 50\% tumor rejection rate for B16shTGF- $\beta$ tumors (Figure $3 \mathrm{H}$ ).

Silencing of tumor-derived VEGF or TGF- $\beta$ prevents Treg recruitment and proliferation in tumor-draining lymph nodes. We analyzed the contribution of Tregs to the growth inhibition of silenced tumors. We first monitored recruitment of total Tregs (Foxp3 ${ }^{+}$among $\mathrm{CD} 4^{+}$cells) and amTregs (CD44 ${ }^{\text {hi }}$ among CD $4^{+} \mathrm{Foxp}^{+}$ cells) in tumor-draining (dLNs) and non-draining (ndLNs) lymph nodes (Figure 4A and Supplemental Figure 5A). Treg proportions progressively increased to greater than $15 \%$ at day 20 after tumor implantation in B16 dLNs compared with ndLNs. In contrast, there was a significantly reduced increase in this proportion in B16-shVEGF and B16-shTGF- $\beta$ dLNs. This effect was more pronounced for the recruitment of amTregs in dLNs.

This decreased recruitment of Tregs correlated with their decreased activation and proliferation. We adoptively transferred ex vivo CFSE-labeled Thy $1.1^{+} T$ cells obtained from naive mice to Thy $1.2^{+}$mice injected with $W T$ or silenced tumors one day earlier (Figure 4B and Supplemental Figure 5B). Ten days later, 30\% to 33\% of the transferred Tregs had undergone several divisions in the $n d L N s$, in line with the high turnover of Tregs at steady state (44). This 

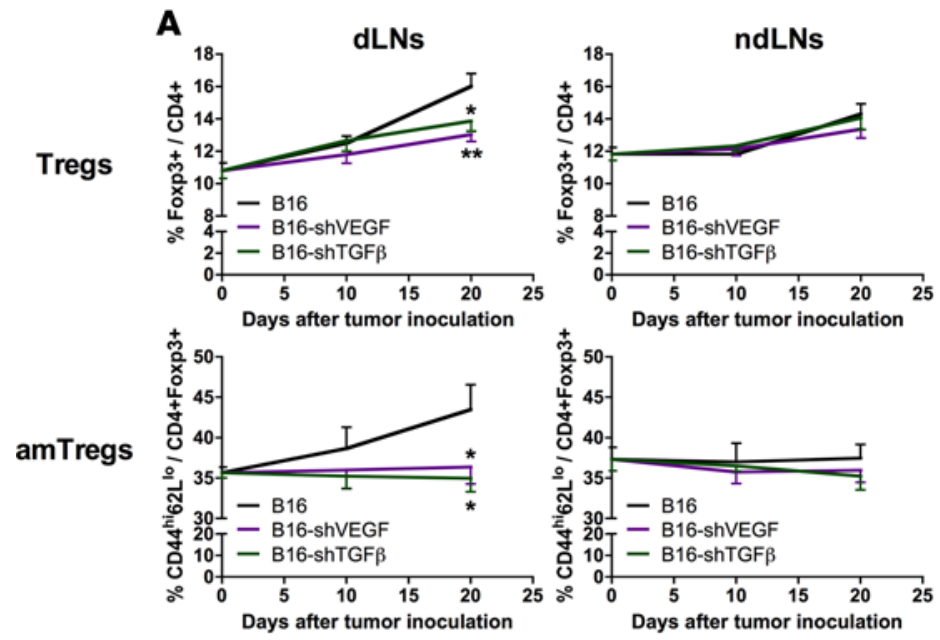

B
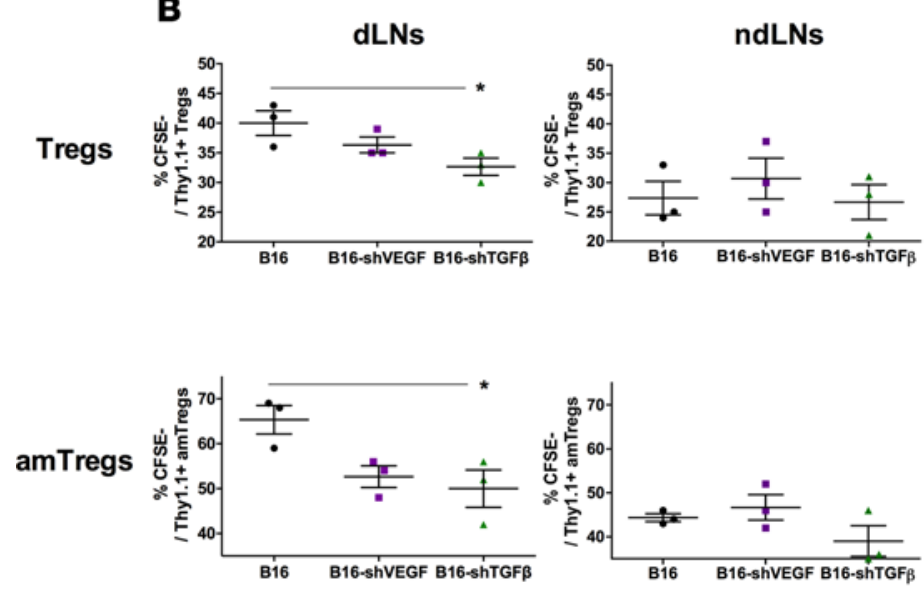
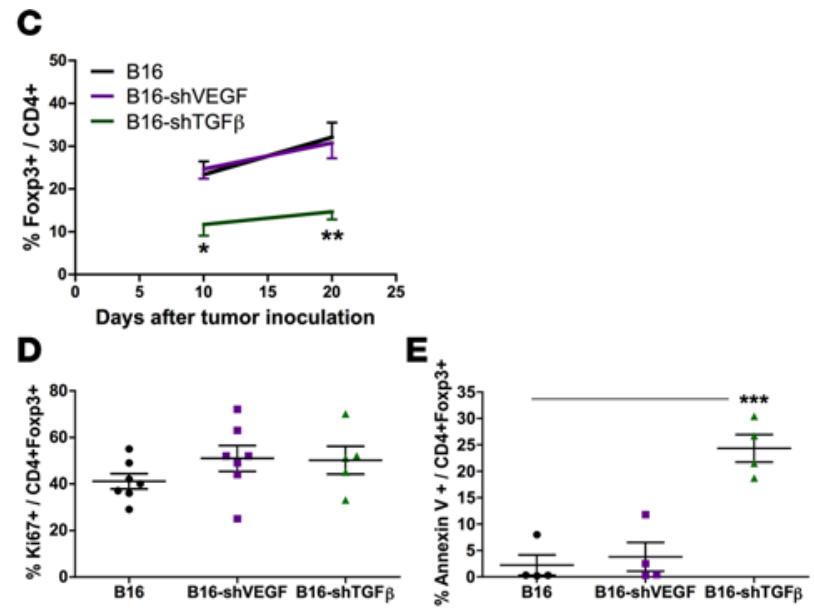

F

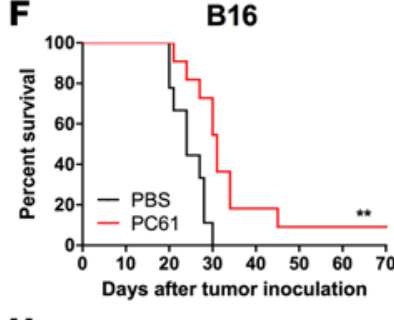

B16

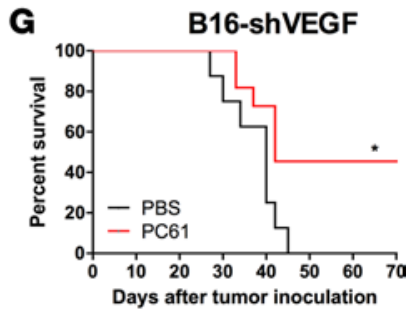

Figure 4. VEGF or TGF- $\beta$ silencing abolishes Treg and activated/memory Treg (amTreg) accumulation and proliferation in the tumor-draining lymph nodes, but only TGF- $\beta$ silencing affects tumor-infiltrating Tregs. (A) Percentages of Tregs among CD4 ${ }^{+}$T cells (upper panels) and CD44 $4^{\text {hi }}$ amTregs (lower panels) in draining lymph nodes (dLNs, left panels) and nondraining lymph nodes (ndLNs, right panels) of tumor-bearing mice, analyzed at the indicated time points after s.c. inoculation of $10^{5}$ WT or silenced B16 tumor cells; $n=12$ mice per group in 3 independent experiments. (B) One-day tumor-bearing WT Thy1.2 mice received CFSE-labeled Thy1.1 congenic cells from naive C57BL/6 mice. Division profiles of Thy1.1+ donor Tregs and amTregs were evaluated by flow cytometry 10 days later. Mean percentages of cells that had undergone at least one division are shown in histograms; $n=3$ mice per group. (C) Kinetic representation of Treg proportions in WT, VEGF- or TGF- $\beta$-silenced tumors after s.c. inoculation in WT mice; $n=8$ mice per group in 2 independent experiments. ( $\mathbf{D}$ and $\mathbf{E})$ Flow cytometric quantification of (D) Ki67 and (E) annexin $\mathrm{V}$ expression in CD4 $4^{+}$Foxp3 ${ }^{+}$ Tregs in WT, VEGF-, or TGF- $\beta$-silenced tumors at day 20; $n=4-7$ mice per group in 2 independent experiments. (F-H) Kaplan-Meier survival curves of C57BL/ 6 WT mice depleted or not of Tregs by anti-CD25 antibody (PC61) and challenged the day after with 105 WT (F), VEGF- (G), or TCF- $\beta-($ H) silenced tumor cells; $n=10$ mice per group in 2 independent experiments. Statistical significance of the survival curves was analyzed by using the log-rank test, and the other results by using the Ordinary one-way ANOVA test with Bonferroni's correction $\left({ }^{*} P<0.05 ;{ }^{*} P<0.005,{ }^{* * *} P<0.001\right)$.

proportion was increased in $d L N$ s of mice harboring B16 tumors, but not in those of mice harboring silenced tumors. These observations were even more striking when looking at am Tregs.

Tumor-derived TGF- $\beta$, but not VEGF, is crucial for Treg recruitment and survival in the tumor mass. We next looked at the dynamics of Treg recruitment within tumors. The percentage of tumor-infiltrating Tregs was higher and increased over time in B16 and B16-shVEGF tumors, reaching approximately $33 \%$ of $\mathrm{CD}^{+}$cells at day 20 (Figure $4 \mathrm{C}$ and Supplemental Figure 5C). In contrast, the percentage of tumor-infiltrating Tregs always stayed around 10\% in B16-shTGF- $\beta$ tumors. This translated into a 4 -fold increase of the Teff/Treg ratio in B16-shTGF- $\beta$ tumors compared with B16 and B16-shVEGF tumors (data not shown).

These differences were not due to the generation of peripherally induced Tregs ( $p$ Tregs), described as largely TGF- $\beta$ dependent (36). Thy 1.1 $1^{+} C D 4^{+} \mathrm{CD} 25^{-} \mathrm{GFP}-\mathrm{Teffs}$ were obtained from naive Thy $1.1^{+}$Foxp3EGFP mice and injected into Thy $1.2^{+}$mice. Upon transfer in $\mathrm{CD} 3 \varepsilon-\mathrm{KO}$ mice, these effector cells trigger an inflammatory response 
A
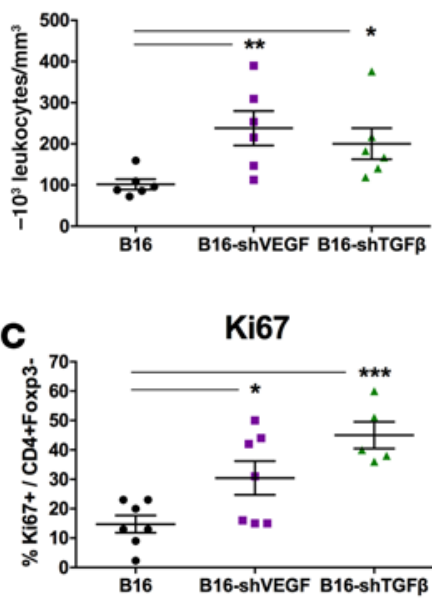

CD8

Teffs
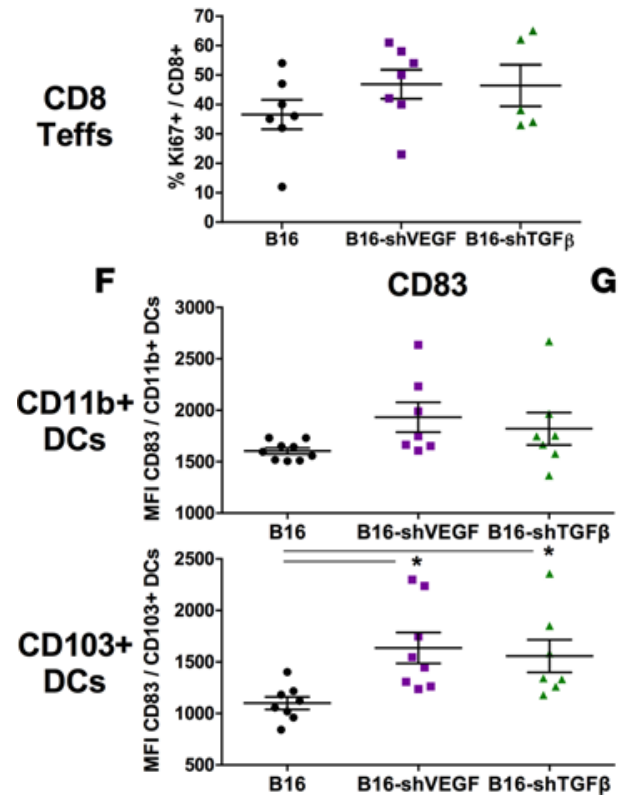

B

B16

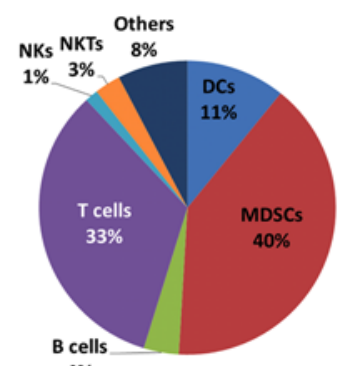

D

$4 \%$
B16-shVEGF

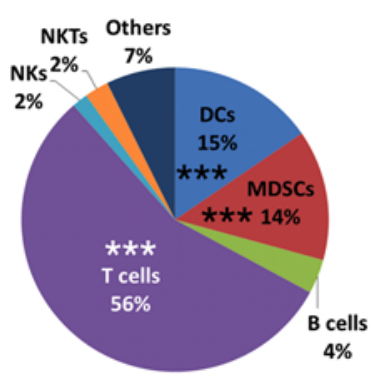

B16-shTGF $\beta$

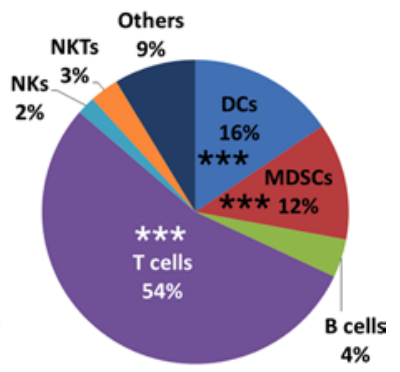

$\mathbf{E}$
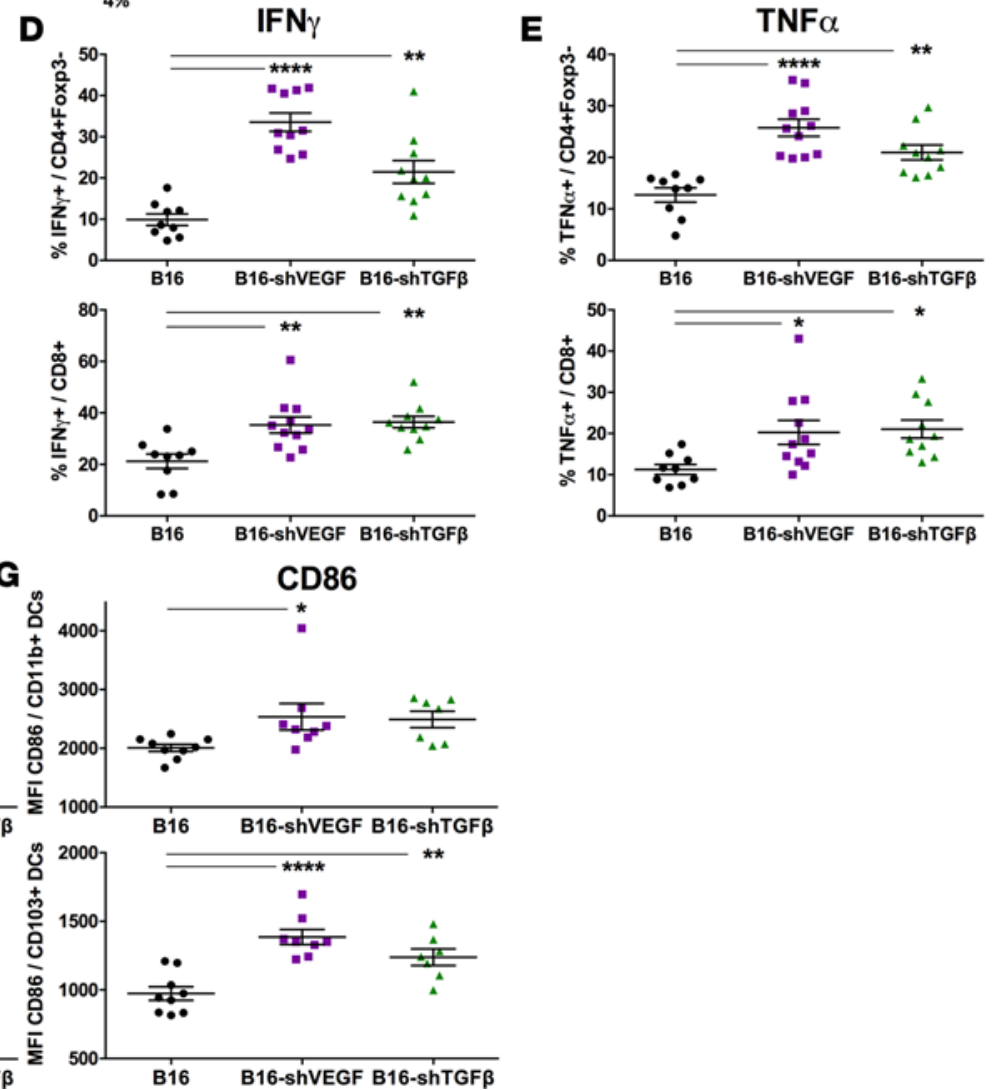

Figure 5. VEGF or TGF- $\beta$ silencing dramatically impacts the composition and activation status of the tumor immune cell infiltrates. (A) Density of tumor infiltration by immune cells at day 20 , calculated as $10^{3} \mathrm{CD}_{4} 5^{+}$cells per $\mathrm{mm}^{3}$ in WT, VEGF-, or TCF- $\beta$-silenced B16 tumors. (B) Pie chart representation of the mean composition of the immune cell infiltrates, including T cells (CD3e+NKp46-), B cells (B220+MHCII+), DCs (CD11c $\left.\mathrm{MHClI}^{+}\right)$, myeloid-derived suppressor cells (MDSCs) (CD11c-MHCII-CD116+Gr-1+), NK cells (NKp46+), NKT cells (CD3e+NKp46+), and "others" (negative for the markers listed above). (C-E) Percentages of $\mathrm{Ki}^{+} 7^{+}(\mathbf{C}), \mathrm{IFN}-\gamma^{+}(\mathbf{D})$, and TNF- $\alpha^{+}(\mathbf{E})$ in $\mathrm{CD}^{+} \mathrm{Foxp}^{-}$(upper panel) and CD8 ${ }^{+}$(lower panel) Teffs in the tumors measured ex vivo (C) or after polyclonal stimulation ( $\mathbf{D}$ and $\mathbf{E}$ ) by flow cytometry. (F) Expression levels of CD83 (left panels) and CD86 (right panels) by CD11b+CD103- (upper panels) and

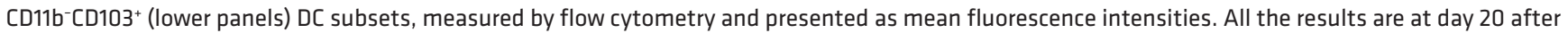
tumor inoculation and for $n=5$ to 10 mice per group in at least 2 independent experiments. Statistical significance of the results was analyzed by using the Ordinary one-way ANOVA test with Bonferroni's correction $\left({ }^{*} P<0.05\right.$; ${ }^{* *} P<0.005$, ${ }^{* * *} P<0.001,{ }^{* * *} P<0.0001$ ).

that is partly controlled by the generation of pTregs (Supplemental Figure 6). In contrast, upon transfer 1 day after the injection of WT or silenced tumors, we could not detect substantial Thy $1.1^{+} \mathrm{CD}^{+} \mathrm{GFP}^{+}$pTregs in dLNs or in tumors (Supplemental Figure 6). Additionally, there were also no statistically significant differences for Ki67 ${ }^{+}$Treg proportions in the different tumors (Figure 4D and Supplemental Figure 5D). In contrast, $25 \%$ of Tregs in B16-shTGF- $\beta$ tumors were positively stained for annexin V, compared with less than 5\% in B16 and B16-shVEGF tumors (Figure 4E and Supplemental Figure 5D). We also analyzed various activation markers of Tregs in the silenced tumors (CTLA-4, Icos, PD-1, and CD69) and did not observe any major changes in their expression across the different groups (data not shown).

To evaluate the functional relevance of Tregs infiltrating WT and B16-shVEGF tumors, we treated mice 
with Treg-depleting anti-CD25 antibodies 1 day prior to tumor challenge (Figure 4, F-H). Compared with PC61-treated B16 tumors, Treg depletion had little effect on B16-shTGF- $\beta$ tumor growth (Figure 4, F and H, $P=0.3589$ ), while it led to an eradication rate of over 50\% for B16-shVEGF tumors (Figure 4G, $P=0.0036$ ).

VEGF or TGF- $\beta$ silencing dramatically impacts the density and composition of tumor-infiltrating hematopoietic cells. We analyzed the composition and activation status of tumor immune infiltrates at day 20 (Figure 5 and Supplemental Figure 7). TGF- $\beta$ or VEGF silencing induced a marked increase in the density of the immune infiltrates (Figure 5A). MDSCs represented 40\% of the total infiltrate in B16 tumors. Their proportion was considerably reduced in B16-shVEGF and B16-shTGF- $\beta$ tumors $(14 \%$ and $12 \%$, respectively). In contrast, DC and $\mathrm{T}$ cell proportions were increased by $40 \%$ and $70 \%$ in B16-shVEGF and B16-shTGF- $\beta$ tumors compared with WT tumors, respectively. The small proportions of granulocytes, B cells, NK cells, and NKT cells present in the tumor infiltrates did not vary between tumors (Figure 5B and Supplemental Figure 7A).

VEGF or TGF- $\beta$ silencing leads to increased activation of tumor-infiltrating Teffs and DCs. Tumor-infiltrating CD8 T cells expressing Ki67 did not vary appreciably between the different tumors. In contrast, the silencings induced a significant increase in the proportions of $\mathrm{CD}^{+} \mathrm{FoxP} 3^{-} \mathrm{Ki}^{-} 7^{+} \mathrm{Teffs}$, which was more pronounced in the B16-shTGF- $\beta$ than in the B16-shVEGF tumors (Figure 5C and Supplemental Figure 7B). Both $\mathrm{CD}^{+}$and $\mathrm{CD} 8^{+}$Teffs from B16-shTGF- $\beta$ and B16-shVEGF tumors had increased production of IFN- $\gamma$ and TNF- $\alpha$ inflammatory cytokines, compared with B16 tumors (Figure 5, D and E and Supplemental Figure 7C). The increase in IFN- $\gamma$ and TNF- $\alpha$ production in $\mathrm{CD}^{+}$Teffs was more upregulated in $\mathrm{CD}^{+}$ Teffs from B16-shVEGF tumors. CD83 and CD86 expression on CD11b ${ }^{+}$and CD103 ${ }^{+}$DCs subsets was increased as well as in B16-shTGF- $\beta$ and B16-shVEGF tumors, compared with B16 tumors (Figure 5F and Supplemental Figure 7D).

Silencing of tumor-derived VEGF or TGF- $\beta$ produces partially nonoverlapping upmodulation of immune responses in the tumor tissue transcriptome and synergizes to induce T cell-dependent tumor eradication. We directly compared the modulated signatures of B16-shTGF- $\beta$ and B16-shVEGF tumors (Figure 6). Already at day 4, among the 605 modulated signatures of either silenced tumors, $377(62 \%)$ were commonly modulated in both B16shTGF- $\beta$ and B16-shVEGF tumors. When looking only at immune-related signatures, 128 of the 163 modulated ones (78\%) were commonly regulated in both B16-shTGF- $\beta$ and B16-shVEGF tumors. At day 14 , the percentages of commonly regulated signatures remained at $62 \%$, while that of immune-related signatures rose to $97 \%$ (Figure $6 \mathrm{~A}$ ). These striking similarities between the pleotropic changes in immune-related signatures suggested a common downstream pathway, which we hypothesized was linked to Treg functions. We thus compared the modulated signatures resulting from VEGF or TGF- $\beta$ silencing to those resulting from Treg depletion. Strikingly, all the upmodulated immune-related signatures of B16-shTGF- $\beta$ and B16shVEGF tumors were also upregulated in WT tumors developing in Treg-depleted mice (Figure 6A).

We then looked at the differently modulated signatures between B16-shTGF- $\beta$ and B16-shVEGF tumors. While VEGF or TGF- $\beta$ silencing led to markedly different regulation of diverse signatures related to metabolic, catabolic, and cell adhesion functions, they led to only limited differences in the modulation of immune-related signatures. There were only 11 immune-related signatures that were differentially regulated after either TGF- $\beta$ or VEGF silencing (Figure 6B). Furthermore, these differences were only quantitative, as all of these signatures were upregulated in both B16-shTGF- $\beta$ and B16-shVEGF tumors compared with B16 tumors.

To investigate whether the co-silencing of TGF- $\beta$ or VEGF could be synergistic, we generated B16-shTGF- $\beta$ / shVEGF tumors (Supplemental Figure 8). Although the in vitro doubling time for the $W T$ and co-silenced tumors was identical, the growth of B16-shTGF- $\beta /$ shVEGF tumors was slightly delayed in CD3E-KO mice (Figure 6C). Strikingly, B16-shTGF- $\beta$ /shVEGF tumor growth was markedly slowed in WT C57BL/ 6 mice, ultimately resulting in a 40\% complete eradication rate without any therapeutic intervention (Figure 6D).

Tumor-derived VEGF and TGF- $\beta$ both contribute to dominant tolerance imprinting. The dominant tolerance of tumors is characterized by their refractoriness to the injection of tumor-specific activated $\mathrm{T}$ cells (17). Given the decreased Treg response to the silenced tumors and the early reprogramming of the tumor environment, we investigated whether VEGF or TGF- $\beta$ silencing could prevent the dominant tolerance imprinting. We engineered our tumors to express the lymphocytic choriomeningitis virus (LCMV) envelope glycoprotein model antigen (gp), and harvested tumor-specific Teffs from immunized WT C57BL/6 mice. Dominant tolerance installation was then assessed by comparing the effect of gp-specific amTeff adoptive transfer at day 0 or day 4 after tumor cell inoculation (Figure 7, A-D). As previously reported (17), B16 tumors became refractory to the transfer of amTeffs at day 4 (Figure 7A). In contrast, VEGF- and/or 
A

Day 4

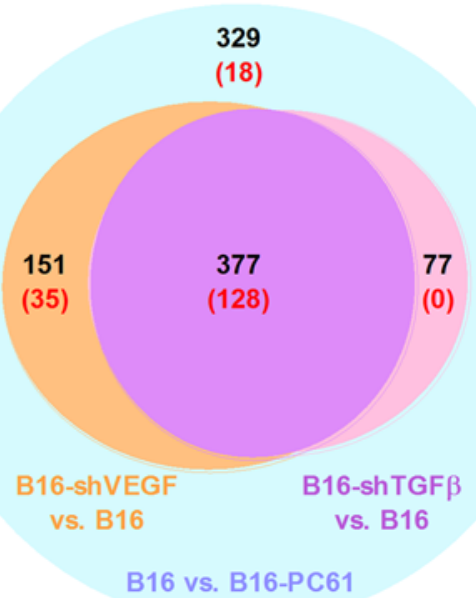

\section{Day 14}

331

(16)

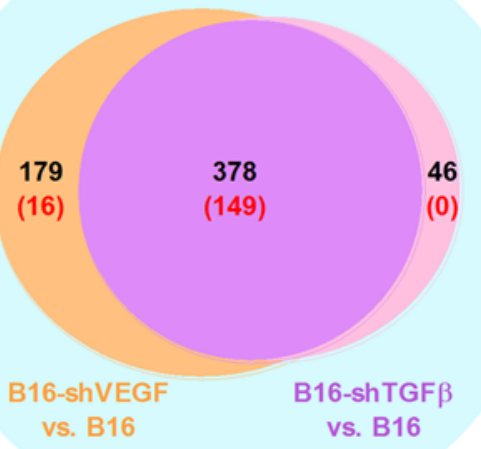

B16 vs. B16-PC61

B

\section{B16-shVEGF vs. B16-shTGF $\beta$}

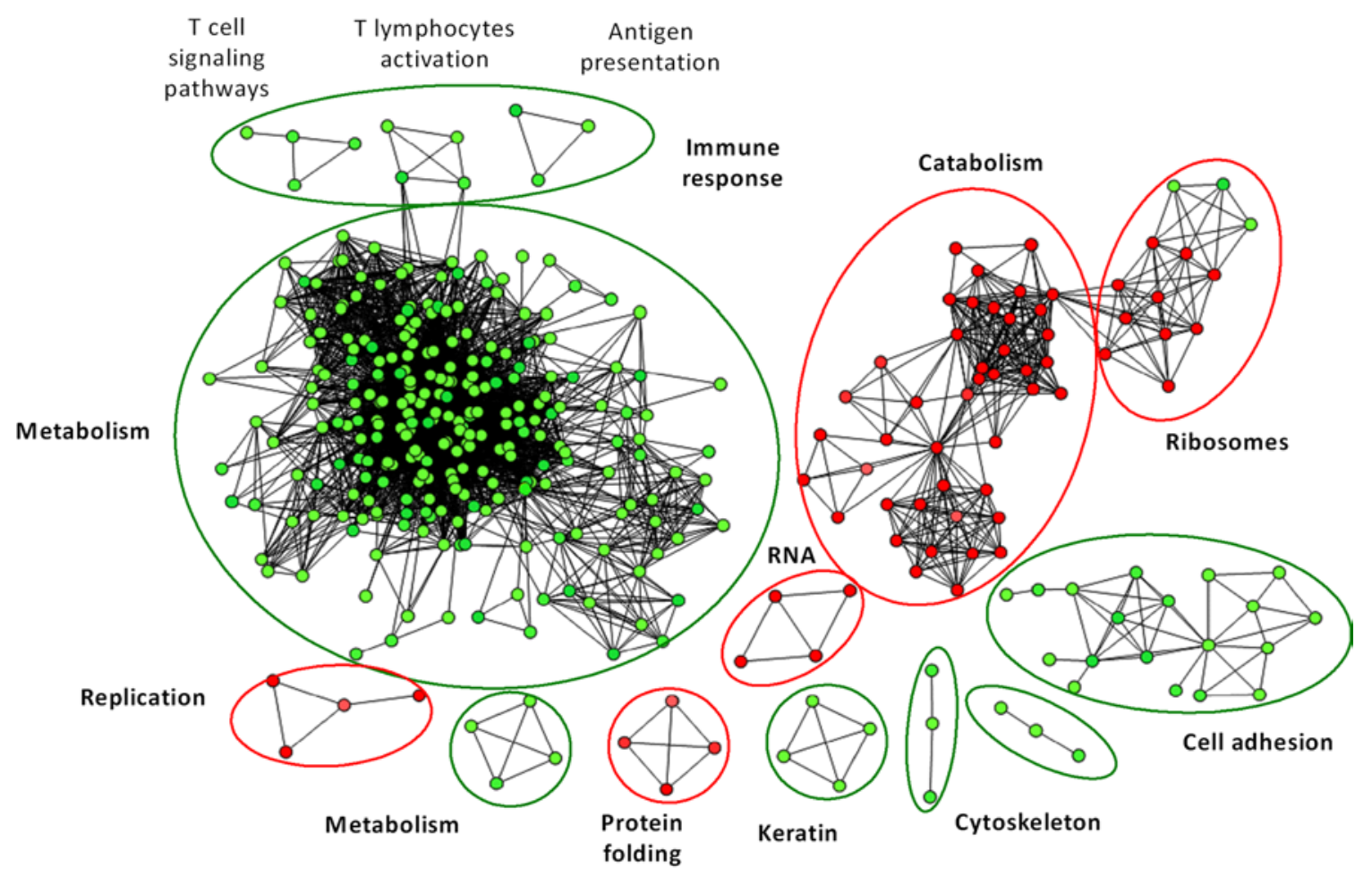

C

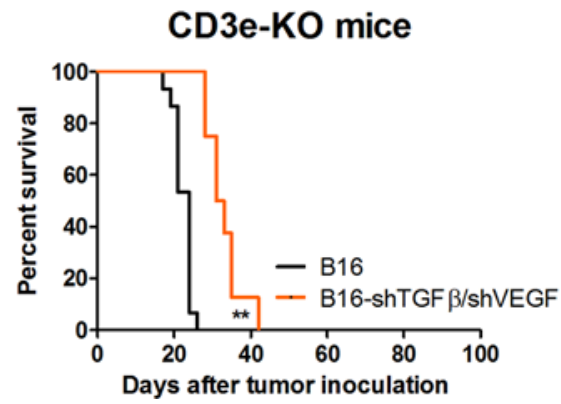

D

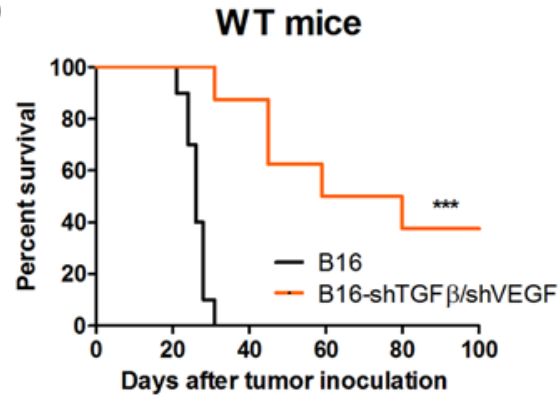


Figure 6. VEGF or TGF- $\beta$ silencing concomitantly induces similar and partially nonoverlapping effects on antitumor immune response that correlate with defects in Treg response, and their co-silencing leads to T cell-dependent spontaneous rejection of B16 tumors. (A) Venn representations of the comparison between significantly modulated signature numbers (in black) measured by microarray analyses in three conditions (B16 vs. B16-PC61 in blue, B16 vs. B16-shVEGF in orange, and B16 vs. B16-shTGF- $\beta$ in purple), 4 days (left panel) or 14 days (right panel) after tumor inoculation. Numbers of immunerelated signatures are represented in red. (B) Cytoscape representation of signatures differently regulated in B16-shVEGF and B16-shTCF- $\beta$ tumors, measured by microarray. (C and D) Kaplan-Meier survival curves of (C) CD3 $\varepsilon-K 0$ and (D) C57BL/6 WT mice inoculated with the listed tumors; $n=10$ mice per group in 2 independent experiments. Statistical significance of the survival curves was analyzed by using the log-rank test $\left({ }^{* *} P<0.005,{ }^{* * *} P<0.001\right)$.

TGF- $\beta$-silenced tumors remained permissive to immune attack by amTeffs at day 4 (Figure 7, B-D).

Combination of VEGF and TGF- $\beta$ inhibitory drugs is synergistic. To explore the therapeutic potential of VEGF and TGF- $\beta$ inhibitors, we first treated established B16 tumors with a combination of existing drugs targeting VEGF or TGF- $\beta$. We selected sunitinib, targeting the VEGF pathway $(26,27)$, and SB431542, an inhibitor of TGF- $\beta$ signaling $(45,46)$. While SB431542 and sunitinib alone induce moderate effects on B16 growth (a respective increase of 1 and 5.5 days of median survival), their combination appears synergistic (Figure 7, E and F) and leads to a significant 10.5 days increase in the median survival (Figure 7F).

Silencing of VEGF and/or TGF- $\beta$ leads to a dramatic improvement of anti-PD-1/anti-CTLA-4 treatment efficacy. To deepen the potential use of VEGF and/or TGF- $\beta$ targeting, we then assessed the impact of anti-PD-1/anti-CTLA-4 antibody immunotherapy on the silenced-tumor growth (Figure 7, G-J). This combined treatment led to a $27 \%$ rejection rate of B16 WT tumors (Figure 7G). In silenced tumors (Figure $7, \mathrm{H}-\mathrm{J}$ ), these complete response rates were significantly increased, reaching up to $80 \%$ in the doubly silenced tumors (Figure 7J).

\section{Discussion}

We identified tumor-produced VEGF and TGF- $\beta$ as molecular drivers of tolerance imprinting by transcriptomic analyses of tumor tissues. Noteworthily, these studies were performed on samples from day 4 after tumor cell inoculation in order to assess early events that could be linked to the imprinting of the tumor tolerant environment (17). At this time point, silenced tumors were hardly detectable. This rendered flow cytometric and histological studies impossible, while transcriptomic studies allowed a global early assessment of the tumor microenvironment. The inactivation of VEGF and TGF- $\beta$ completely changes the immune infiltrate of tumors from a tolerant-oriented one to an effector-oriented one. Likewise, it unleashes the potential of natural tumor-specific effector immune responses that result in a marked delay in tumor growth in poorly immunogenic tumors and even in tumor eradication in more immunogenic tumors. Finally, VEGF and TGF- $\beta$ inactivation changes the tumor environment from refractory to permissive in cellbased immunotherapies and dramatically improves the efficiency of checkpoint inhibitors.

The global transcriptomic studies that assessed the effects of VEGF and TGF- $\beta$ silencing produced molecular signatures discovered using unsupervised methods, and are thus robust. Furthermore, we showed that such signatures, obtained from mouse data sets, have the power to discriminate clinical samples (i.e., metastases from primary melanoma). Although the information related to the human data set do not allow interpreting these results, they strongly reinforce the relevance of our transcriptomic studies.

It is surprising that two cytokines with largely different functions have such remarkably similar effects in the context of tumors. Indeed, transcriptomic analyses of shTGF- $\beta$ and shVEGF tumors show a major pleiotropic impact of VEGF and TGF- $\beta$ on immune responses, from lymphocyte recruitment to antigen presentation and lymphocyte activation. Comparison of the signatures commonly or differently upregulated after VEGF or TGF- $\beta$ silencing highlights similarities rather than differences. There are 91 immune-related signatures that are similarly upregulated in response to either silencing, while only 11 are differently regulated. Closer examination of the latter showed that they are composed of genes related to TCR signaling (such as CD3e, CD38, CD81, Zap70, or Lck), T cell activation (e.g., CD28, TNF- $\alpha$, ADAM17, IL-4, IL-7, IL-15, IL-18) and antigen presentation (e.g., MHC molecules, CD83, CD86) (data not shown). Moreover, their differential regulation is only quantitative; i.e., higher upregulation in response to TGF- $\beta$ silencing.

Such similarities strongly suggest that the effects of these cytokines are related to a common downstream effect, which we showed is linked to Tregs, although differently. TGF- $\beta$ inactivation had a largely direct effect on Tregs, with decreased tumor infiltration and increased apoptosis, in line with its known properties $(34,37,47)$. In contrast, the effect of VEGF on Treg appears more indirect. By affecting the maturation of DCs and, possibly more importantly, by modifying the metabolic environment of tumors, VEGF 
A

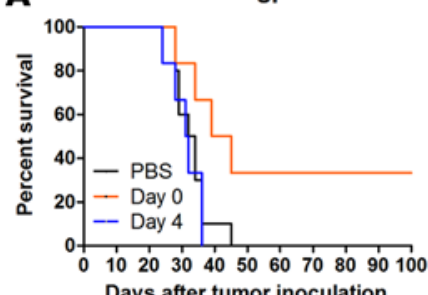

Days after tumor inoculation

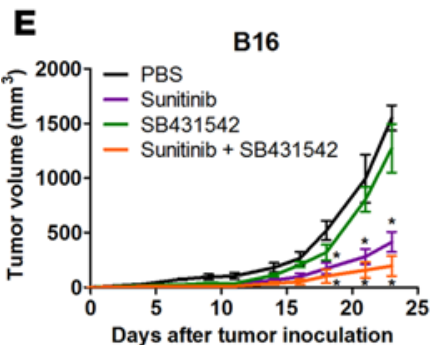

B

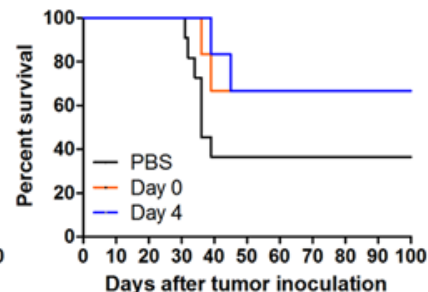

$\mathbf{F}$
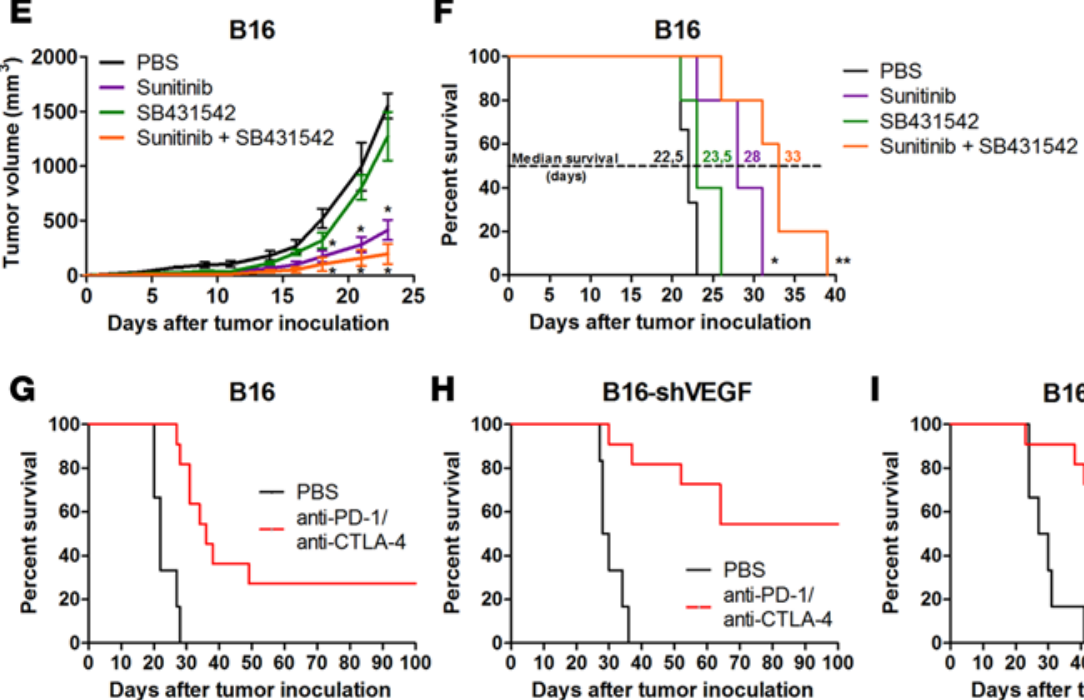

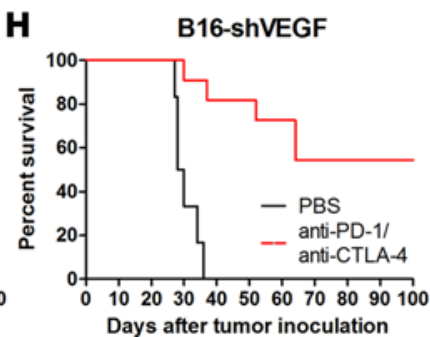

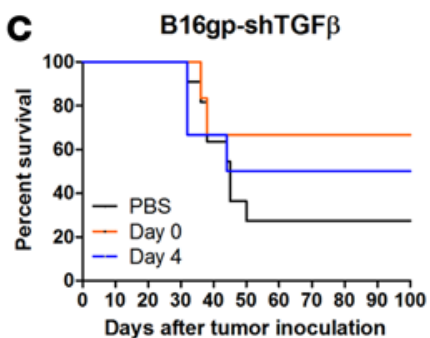

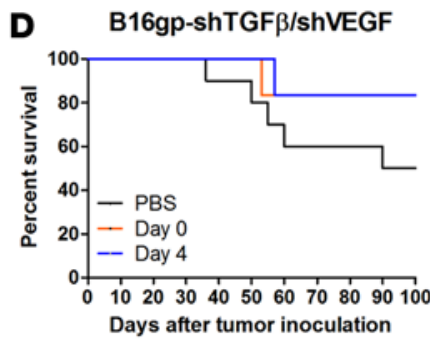

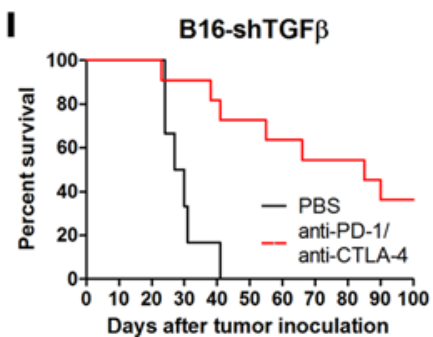

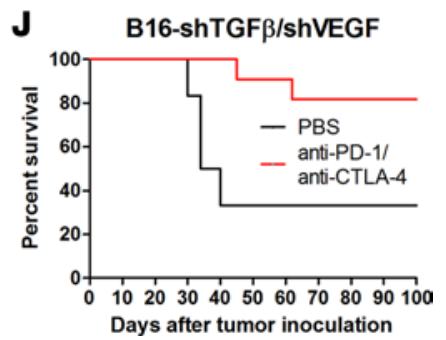

Figure 7. Tumor-derived VEGF and TGF- $\beta$ contribute to the onset of dominant tolerance and are therefore valuable targets for combination therapies. (A-D) Kaplan-Meier survival curves of B16gp- (A), B16gp-shVEGF- (B), B16gp-shTCF- $\beta$ - (C), or B16gp-shTCF- $\beta /$ shVEGF- (D) bearing WT C57BL/6 mice injected s.c. with recombinant lymphocytic choriomeningitis virus envelope glycoprotein (gp) model antigen-expressing tumors at day 0 and i.v. with PBS or with $4 \times 10^{6} \mathrm{gp}$-specific activated/memory effector T cells (amTeffs) from immunized mice at day 0 or day $4 ; n=5$ mice per group. Survival rate was significantly higher in B16gp-bearing mice when amTeffs were injected at day 0 compared with day 4 (log-rank test, $P=0.0324$ ), whereas it was not in response to the other tumors. (E) Kinetics and (F) Kaplan-Meier survival curves of parental B16 tumor-bearing WT C57BL/6 mice treated or not with sunitinib during 2 weeks and/or SB431542 at days 3, 6, and $9 ; n=5$ per group. (G-J) Kaplan-Meier survival curves of B16- (G), B16-shVEGF- (H), B16shTGF- $\beta$ - (I), or B16-shTCF- $\beta /$ shVEGF- (J) bearing WT C57BL/6 mice treated or not at days 3, 6, and 9 with a cocktail of $100 \mu \mathrm{g}$ anti-CTLA-4 and $250 \mu \mathrm{g}$ anti-PD-1 antibodies; $n=11$ mice per group in 2 independent experiments. Survival rate was significantly higher in the B16-shVEGF-bearing $(P=0.048)$ and B16-shTGF- $\beta$ /shVEGF-bearing (log-rank test, $P=0.0041$ ) treated groups compared with the B16-bearing treated group. Statistical significance of the survival curves was analyzed by using the log-rank test $\left({ }^{*} P<0.05 ;{ }^{* *} P<0.005\right)$.

may differently alter the fitness of Treg and Teffs (28). We confirmed these observations by showing that the very same immune-related signatures upregulated in the transcriptomes of TGF- $\beta$ - or VEGF-silenced tumors are similarly upregulated in the tumors growing in Treg-depleted hosts. Thus, TGF- $\beta$ may primarily reduce the number of Tregs while VEGF may primarily reduce their fitness. The resulting common Treg "insufficiency" explains the observed similarities. These two different mechanisms - mostly quantitative and mostly qualitative - are supported by the cooperativity of the effects mediated by the 2 cytokines. Their co-silencing improves the complete response rate from $0 \%$ in WT tumors and singly silenced tumors to $40 \%$ for poorly immunogenic tumors, and up to $60 \%-80 \%$ in immunogenic tumors. Noteworthily, the immune responses unleashed by these effects on Tregs appear in large part to be mediated by a similar (a) decrease in the proportion and number of MDSC, and (b) increase in DCs and Teff activation.

The recent success of checkpoint inhibitor-based immunotherapies of cancer $(9,10)$ has highlighted the potential of the natural effector immune response against cancer. By efficiently boosting Teffs, these effectors can penetrate and destroy tumors. However, it has also appeared that these therapies have an important effect on modifying the metabolic environment of tumors, at least in part explaining their effects. As these therapies are only partially effective (i.e., do not always achieve a complete response, nor are they effective in all patients), there is a need to improve them. This is likely to be achieved by combinatorial treatments (48). Here we report that the combination of anti-TGF- $\beta$ and anti-VEGF drugs is synergistic, providing a 
rationale to test their therapeutic combination in patients. Combinations of VEGF- or TGF- $\beta$-inhibitory molecules with other therapeutic regimens have already been tested. They revealed that TGF- $\beta$ targeting improves the efficacy of diverse radiotherapeutic and chemotherapeutic protocols, as well as immunotherapies using vaccination strategies or anti-OX40 antibodies. These positive effects have been linked to effects on stroma normalization, transport of therapeutic molecules into the tumor site, and modification of the intratumoral Teff/Treg balance (49). VEGF inhibition has been combined with a much broader range of therapeutic products. These include radiotherapeutic and chemotherapeutic agents, $\mathrm{T}$ cell-based cell therapies, immunostimulatory cytokines such as IL-12, IL-21, and IFN- $\alpha$, and immunomodulatory antibodies including anti-41BB, anti-PD-1, and anti-CTLA-4 (50). In all cases, VEGF inhibition has been associated with improved antitumor effects of these therapies. Interestingly, a combination of bevacizumab (antiVEGF) and ipilimumab (anti-CTLA-4) in metastatic melanoma patients led to increased Teff infiltration in the tumor and improved tumor outcome compared with ipilimumab alone (51). Similar results were obtained in mice using a combination of anti-VEGF and anti-PD-1 antibodies (27).

Altogether, our results provide additional evidence that Tregs are the main players in dictating the tolerant tumor environment and must be controlled to improve immunotherapies. We identify tumor-produced VEGF and TGF- $\beta$ as the main molecular controllers of the Treg response. Their inhibition changes the tumor environment towards Teffs from a refractory one to a permissive one. Likewise, they identify these molecules as excellent candidates for immunotherapies of cancer that combine therapies disrupting the tolerant environment with biotherapies providing better effectors.

\section{Methods}

Animals and transplanted tumor models. Female BALB/cJRj and C57BL/6JRj mice (6-8 weeks old) were obtained from JANVIER SAS. Thy-1.1 C57BL/6 mice, CD3 $\varepsilon-\mathrm{KO}$ mice, and Foxp3-EGFP-Thy-1.1 mice were maintained in our animal facility. Mice were housed in filter-topped cages under specific pathogen-free conditions. All mice were treated in accordance with the European Union guidelines for animal experimentation. Mycoplasma-free B16F10 tumor cells were obtained from the ATCC (catalog CRL-6475) and cultured in DMEM (Invitrogen) complemented with 10\% FCS, 2 mM L-glutamine (Invitrogen), and 100 $\mathrm{U} / \mathrm{ml}$ penicillin-streptomycin (Invitrogen). The mycoplasma-free AB1-HA mesothelioma cell line, which expresses the murine influenza HA (52), was supplied by B. Scott (Centre for Functional Genomics and Human Disease, Monash Institute of Medical Research, Clayton, Victoria, Australia) and cultured in RPMI (Invitrogen) complemented with 10\% FCS, $2 \mathrm{mM} \mathrm{L}$-glutamine, and $100 \mathrm{U} / \mathrm{ml}$ penicillin-streptomycin. For in vivo experiments, $10^{5} \mathrm{~B} 16 \mathrm{~F} 10$ or $5 \times 10^{5} \mathrm{AB} 1-\mathrm{HA}$ tumor cells were injected s.c. in the right flank or i.v. in the retroorbital sinus of the mice. Tumor volume was determined by measuring perpendicular tumor diameters $\mathrm{L}$ and 1 using Vernier calipers, calculated as $(\mathrm{L} \times 12) / 2$, and expressed as $\mathrm{mm}^{3}$. The right inguinal $\mathrm{LN}$ was used as the dLN. The left axillary LN was used as the ndLN. Survival was determined by the time at which mice required euthanasia, when tumor volume reached $1,500 \mathrm{~mm}^{3}$. Tumor in vitro doubling time was calculated using Roth V. 2006 Doubling Time Computing (http://www.doubling-time.com/compute.php).

Plasmid and viral vectors. Vectors encoding shRNA were from Sigma-Aldrich (pLKO.1 puro vectors: Vegf-a: NM-009505.2-592s1c1 TCRN0000066818 TRC1; Tgf- $\beta 1$ : NM-011577.1-1753s1c1 TCRN0000065993 TRC1). They also contained the puromycin resistance gene, which was used to select transduced cells. The VEGF-specific shRNA-carrying vector used to create the co-silenced cells is the pLKO.1 plasmid from SigmaAldrich in which we replaced the puromycin resistance gene with the neomycin resistance gene. The expression vector for the gp model antigen derived from the LCMV virus, encapsulable by lentiviruses and permitting gp protein and GFP expression, is derived from a vector previously used in our laboratory (53). Plasmids were amplified in E. coli INV110 bacteria, purified with a Nucleobond Xtra Maxi EF kit (Macherey-Nagel), and their yield was assessed with a NanoDrop 1000 spectrophotometer (NanoDrop Products, Thermo Fisher Scientific) and then verified by enzymatic digestion. To produce the lentiviral vectors, a mixture of three plasmids (7.5 $\mu$ g of VSVG, $17.25 \mu \mathrm{g}$ of CMV9, and $21.5 \mu \mathrm{g}$ of shRNA or gp expression plasmids) was precipitated and used to transfect HEK-293T cells as previously described (54). The supernatant of these transfected cells was then sterile filtered using Stericup (Millipore) and concentrated using Centricons (Millipore).

Cell transduction by lentiviruses. Tumor cell transduction was performed by infecting target cells with the viruses for 2 hours in polybrene-containing FBS-free medium before the addition of complete medium. Two days later we replaced the medium with antibiotic-containing medium to select for transduced cells. Antibiotics used were puromycin (Invivogen, ant-pr-1) at $5 \mu \mathrm{g} / \mathrm{ml}$ and G418 (neomycin analog; Invivogen, 
ant-gn-1) at $5 \mathrm{mg} / \mathrm{ml}$. Antibiotic-resistant cells were then cloned by the limiting dilution method.

Immunization and transfer of gp-specific Teffs. C57BL/6 WT mice were immunized twice s.c. at the base of the tail with a lysate of $2 \times 10^{6}$ gp-expressing B16 cells per mouse, prepared as described elsewhere (55) and adjuvanted in $500 \mu \mathrm{g}$ of alum (Thermo Fisher Scientific), 3 and 2 weeks before sacrifice. Gp-specific Teffs were enriched from lymphoid organs by magnetic cell separation (Miltenyi) using biotinylated antiB220, anti-CD25, and anti-Ter119 antibodies (BD Biosciences) to deplete B cells, Tregs, and red blood cells, respectively. Enrichment was checked by FACS and Teffs were then i.v. transferred into C57BL/6 WT receiver mice, 4 days after or on the same day as tumor inoculation.

In vivo depletion of $C D 4^{+} C D 25^{+} T$ cells. Treg ablation was performed by i.p. injection of $125 \mu \mathrm{g}$ of the anti-CD25 mAb PC61 1 day before tumor challenge. This induces a greater than $80 \%$ transient depletion of LN CD25 $5^{\text {hi }}$ cells for approximately 4 weeks in normal mice (56).

Antibodies and flow cytometric analyses. Cells from peripheral LNs (dLN and ndLN) and tumors were obtained by mechanical dissociation and enzymatic digestion (collagenase IV [Sigma-Aldrich] and DNase I [Roche], respectively, as previously described in refs. 44 and 57). Cells were then stained with saturating amounts of various fluorescently labeled antibody combinations including: anti-CD45 (30-F11 clone), antiCD3e (145-2C11 clone), anti-CD4 (RM4-5 clone), anti-CD8 (53-6.8 clone), anti-CD25 (PC61 clone), antiFoxp3 (FJK-16s clone), anti-CD44 (IM7 clone), anti-CD62L (MEL-14 clone), anti-Ki67 (35/KI-67 clone), annexin V, anti-IFN- $\gamma$ (XMG1.2 clone), anti-TNF- $\alpha$ (MP6-XT22 clone), anti-CD11b (M1/70 clone), antiGr1 (Gr-1 clone), anti-B220 (RA3-6B2 clone), anti-NKp46 (29A.14 clone), anti-CD11c (HL3 clone), antiIA/IE (M5/114.15.2 clone), anti-CD83 (Michel-19 clone), and CD86 (GL1 clone) (all from eBioscience or BD Biosciences). Lymphocytes were analyzed with LSR-II (BD Biosciences), 1 to $3 \times 10^{6}$ events were acquired from each tube. Further analyses were performed with FlowJo software (Tree Star).

CFSE staining and adoptive transfer of cells. Cells were labeled with CFSE (Sigma-Aldrich) as described previously (17) and transferred i.v. to 1-day tumor-bearing mice. Adoptive transfer was performed with either (a) unpurified cells from Thy1.1 congenic mice that were further analyzed after transfer into Thy 1.2 mice based on Foxp3 and Thy1.1 expression to identify donor Tregs, or (b) CD4 ${ }^{+} \mathrm{CD} 25^{-} \mathrm{GFP}^{-}$cells from LNs and spleens of Foxp3-EGFP-Thy1.1 congenic C57BL/6 mice which were purified using a FACSAria flow cytometer (BD Biosciences) as previously described (17).

$V E G F$ and TGF- $\beta$-specific $q R T-P C R$ assays. Total RNA was prepared from in vitro tumor cells using the RNeasy Plus Mini Kit (QIAGEN). RNA yield was assessed using the NanoDrop 1000 spectrophotometer. RNA integrity was assessed using an Agilent Bioanalyzer showing a quality RNA integrity number of 8-10 (Agilent Technologies). RT-PCR was performed to synthesize cDNA for PCR analysis. cDNA in each sample was then quantified by qPCR (Applied Biosystems). The primers used for the first amplification of the housekeeping gene (HPRT1) were forward 5'-GACCGGTCCCGTCATGC-3' and reverse 5'-TCATAACCTGGTTCATCATCGC-3'. The primers used for the first amplification of VEGF-A and TGF- $\beta 1$ were obtained from Thermo Fisher: VEGF-A (catalog Mm01281449) and TGF- $\beta 1$ (catalog Mm01178820). qPCR was performed twice using TaqMan Universal PCR master mix (Applied Biosystems).

Transcriptomic experiments and analysis. Tumor microenvironments were collected from punch biopsies (2.5-mm diameter) centered on the inoculation site, thus collecting both tumor and surrounding tissue. Controls originated from naive mouse skin. Samples were incubated overnight in RNAlater (QIAGEN) at $4^{\circ} \mathrm{C}$ and then transferred to $-80^{\circ} \mathrm{C}$ for storage. Samples were lysed and homogenized using the TissueLyser (QIAGEN) and total RNA was purified using Trizol (Invitrogen) according to the manufacturer's instructions. RNA yield was assessed using the NanoDrop 1000 spectrophotometer. RNA integrity was assessed using the Agilent Bioanalyzer showing a quality RNA integrity number of 8-10. The RNA was processed using the Illumina TotalPrep RNA Amplification Kit Protocol according to the manufacturer's protocol. Briefly, labeled complementary RNAs (cRNA) were hybridized overnight to Illumina MouseWG-6 v2.0 Expression BeadChip microarrays. The arrays were then washed, blocked, stained, and scanned on an Illumina BeadStation following the manufacturer's protocols. Illumina BeadStudio software (Illumina) was used to generate signal intensity values from the scans. All mouse-based data sets were deposited in the GEO repository (GSE73384 and GSE68454) and the public human-based data set is freely available (GSE8401). Genes were filtered out from the analysis if their expression was below the detection limit $(P<0.05)$ in at least 2 out of 3 samples in both tumor microenvironment and control groups. Next, data were normalized according to the quantile method using the limma $\mathrm{R}$ package and then log-transformed. The limma package, freely available at http:// 
www.bioconductor.org/packages/release/bioc/html/limma.html, was used to identify differentially expressed genes (Benjamini-Hochberg corrected $P$ value $<0.05$ ) at days 4 and 14 . Hierarchical clustering was performed using Euclidean distance and the Ward agglomeration method. ICA, described elsewhere (43), was used to generate molecular signatures specific for our data sets. GSEA was used in order to test the statistical relevance of Gene Ontology-based and ICA-based molecular signatures in data sets. A detailed explanation of GSEA can be found in Subramanian et al. (58). Overlaps between significantly enriched signatures from GSEA could be visualized in Cytoscape (41) (version 2.8.3) with the Enrichment Map plugin (42). Enrichment Map produced networks whose nodes represent signatures and whose edges represent mutual overlap. This groups highly redundant signatures together as modules. Only gene sets with an FDR $P$ value of less than 0.01 were selected and the mutual overlap coefficient between signatures was set at 0.8 . Modules of functionally related signatures were manually circled and assigned a label. The functional network was manually curated to remove modules containing fewer than 3 signatures, resulting in a simplified network map. Venn representations were performed using the VennDiagram package on the R platform.

Treatments. Sunitinib (Sutent, from Pfizer) was administered by oral gavage at $40 \mathrm{mg} / \mathrm{kg}$, starting 2 days after tumor inoculation and during two weeks. SB431542 (Sigma-Aldrich) was administered by i.p injection at $3 \mathrm{mg} / \mathrm{kg}$ at days 3, 6, and 9 after tumor inoculation. Sunitinib and SB431542 were dissolved in DMSO and diluted in PBS before administration. Antibody-mediated immunotherapy targeting checkpoint inhibitors was performed as described elsewhere (9). Briefly, 3, 6, and 9 days after inoculation, tumor-bearing mice received i.p. a cocktail containing $100 \mu \mathrm{g}$ of anti-CTLA-4 antibody (9D9 clone from BioXcell, catalog BE0164) and $250 \mu \mathrm{g}$ of anti-PD-1 antibody (RPM1-14 clone from BioXcell, catalog BE0146).

Statistics. Statistical analyses of Kaplan-Meier survival curves were performed using the log-rank test. Unless otherwise indicated, statistical significance between independent values was evaluated using an Ordinary one-way ANOVA test with Bonferroni's correction for multiple comparisons. A $P$ value below $0.05\left({ }^{*} P<0.05 ;{ }^{* *} P<0.005,{ }^{* *} P<0.001,{ }^{* * * *} P<0.0001\right)$ was considered significant. Statistical analyses were performed with Prism software (version 6; GraphPad Software Inc.). Data represent the mean \pm SEM.

Study approval. The present studies in animals were reviewed and approved by the French Agricultural Ministry - Animal Welfare Services (Paris, France, accreditation number B75-13-08).

\section{Author contributions}

DK conceived, designed, and supervised the study and obtained funding. TC, DNB, BL, FB, LF, and TV acquired the data. TC, DNB, and DK analyzed and interpreted the data. TC and DNB performed the statistical analyses. BL and FB provided administrative, technical, and material support. TC and DK drafted the manuscript. DNB and BB critically revised the manuscript for important intellectual content.

\section{Acknowledgments}

We thank Christelle Enond, Flora Issert, Olivier Bregerie, and Bocar Kane (Centre d'Exploration Fonctionnelle, Université Pierre et Marie Curie) for animal care and Bruno Gouritin for cell sorting by flow cytometry.

This work was supported by a grant from the Institut National du Cancer, from French state funds within the Investissement d'Avenir programme (ANR-11-IDEX-0004-02; LabEx Transimmunom), and from the European Research Council Advanced Grant (ERC-2012-AdG, TRiPoD, Agreement number 322856) (to D. Klatzmann). T. Courau was partly supported by La Ligue contre le Cancer.

Address correspondence to: David Klatzmann; GHPS 83 Boulevard de l'Hôpital, Bâtiment CERVI, 2ème étage, 75013 Paris, France. Phone: 33.1.42.17.74.61; E-mail: david.klatzmann@upmc.fr.

1. Grivennikov SI, Greten FR, Karin M. Immunity, inflammation, and cancer. Cell. 2010;140(6):883-899.

2. Zitvogel L, Apetoh L, Ghiringhelli F, André F, Tesniere A, Kroemer G. The anticancer immune response: indispensable for therapeutic success? J Clin Invest. 2008;118(6):1991-2001.

3. Billiard F, et al. Regulatory and effector T cell activation levels are prime determinants of in vivo immune regulation. J Immunol. 2006;177(4):2167-2174. 
4. Shimizu J, Yamazaki S, Sakaguchi S. Induction of tumor immunity by removing $\mathrm{CD} 25^{+} \mathrm{CD} 4^{+} \mathrm{T}$ cells: a common basis between tumor immunity and autoimmunity. J Immunol. 1999;163(10):5211-5218.

5. Onizuka S, Tawara I, Shimizu J, Sakaguchi S, Fujita T, Nakayama E. Tumor rejection by in vivo administration of anti-CD25 (interleukin-2 receptor alpha) monoclonal antibody. Cancer Res. 1999;59(13):3128-3133.

6. Galon J, et al. Type, density, and location of immune cells within human colorectal tumors predict clinical outcome. Science. 2006;313(5795):1960-1964.

7. Curiel TJ, et al. Specific recruitment of regulatory T cells in ovarian carcinoma fosters immune privilege and predicts reduced survival. Nat Med. 2004;10(9):942-949.

8. Nishikawa H, Sakaguchi S. Regulatory T cells in tumor immunity. Int J Cancer. 2010;127(4):759-767.

9. Curran MA, Montalvo W, Yagita H, Allison JP. PD-1 and CTLA-4 combination blockade expands infiltrating T cells and reduces regulatory T and myeloid cells within B16 melanoma tumors. Proc Natl Acad Sci U S A. 2010;107(9):4275-4280.

10. Wolchok JD, et al. Nivolumab plus ipilimumab in advanced melanoma. N Engl J Med. 2013;369(2):122-133.

11. Topalian SL, Drake CG, Pardoll DM. Immune checkpoint blockade: a common denominator approach to cancer therapy. Cancer Cell. 2015;27(4):450-461.

12. Johnston RJ, et al. The immunoreceptor TIGIT regulates antitumor and antiviral CD8(+) T cell effector function. Cancer Cell. 2014;26(6):923-937.

13. Wang X, et al. IL-36 $\gamma$ transforms the tumor microenvironment and promotes type 1 lymphocyte-mediated antitumor immune responses. Cancer Cell. 2015;28(3):296-306.

14. Facciabene A, Motz GT, Coukos G. T-regulatory cells: key players in tumor immune escape and angiogenesis. Cancer Res. 2012;72(9):2162-2171.

15. Ondondo B, Jones E, Godkin A, Gallimore A. Home sweet home: the tumor microenvironment as a haven for regulatory T cells. Front Immunol. 2013;4:197.

16. Nehar-Belaid D, Courau T, Dérian N, Florez L, Ruocco MG, Klatzmann D. Regulatory T cells orchestrate similar immune evasion of fetuses and tumors in mice. J Immunol. 2016;196(2):678-690.

17. Darrasse-Jèze G, et al. Tumor emergence is sensed by self-specific CD44hi memory Tregs that create a dominant tolerogenic environment for tumors in mice. J Clin Invest. 2009;119(9):2648-2662.

18. Motz GT, Coukos G. Deciphering and reversing tumor immune suppression. Immunity. 2013;39(1):61-73.

19. Rabinovich GA, Gabrilovich D, Sotomayor EM. Immunosuppressive strategies that are mediated by tumor cells. Annu Rev Immunol. 2007;25:267-296.

20. Johnson BF, Clay TM, Hobeika AC, Lyerly HK, Morse MA. Vascular endothelial growth factor and immunosuppression in cancer: current knowledge and potential for new therapy. Expert Opin Biol Ther. 2007;7(4):449-460.

21. Tian M, Neil JR, Schiemann WP. Transforming growth factor- $\beta$ and the hallmarks of cancer. Cell Signal. 2011;23(6):951-962.

22. Olsson A-K, Dimberg A, Kreuger J, Claesson-Welsh L. VEGF receptor signalling - in control of vascular function. Nat Rev Mol Cell Biol. 2006;7(5):359-371.

23. Gabrilovich DI, et al. Production of vascular endothelial growth factor by human tumors inhibits the functional maturation of dendritic cells. Nat Med. 1996;2(10):1096-1103.

24. Nagaraj S, et al. Regulatory myeloid suppressor cells in health and disease. Cancer Res. 2009;69(19):7503-7506.

25. Li B, et al. Vascular endothelial growth factor blockade reduces intratumoral regulatory $\mathrm{T}$ cells and enhances the efficacy of a GM-CSF-secreting cancer immunotherapy. Clin Cancer Res. 2006;12(22):6808-6816.

26. Terme M, et al. VEGFA-VEGFR pathway blockade inhibits tumor-induced regulatory T-cell proliferation in colorectal cancer. Cancer Res. 2013;73(2):539-549.

27. Voron T, et al. VEGF-A modulates expression of inhibitory checkpoints on CD $8^{+} \mathrm{T}$ cells in tumors. J Exp Med. 2015;212(2):139-148.

28. Chang $\mathrm{CH}$, et al. Metabolic competition in the tumor microenvironment is a driver of cancer progression. Cell. 2015;162(6):1229-1241.

29. Siska PJ, Rathmell JC. T cell metabolic fitness in antitumor immunity. Trends Immunol. 2015;36(4):257-264.

30. Huang $\mathrm{H}$, et al. VEGF suppresses T-lymphocyte infiltration in the tumor microenvironment through inhibition of NF- $\mathrm{kB}$ induced endothelial activation. FASEB J. 2015;29(1):227-238.

31. Bierie B, Moses HL. Tumour microenvironment: TGFbeta: the molecular Jekyll and Hyde of cancer. Nat Rev Cancer. 2006;6(7):506-520.

32. Li MO, Sanjabi S, Flavell RA. Transforming growth factor-beta controls development, homeostasis, and tolerance of T cells by regulatory T cell-dependent and -independent mechanisms. Immunity. 2006;25(3):455-471.

33. Adeegbe DO, Nishikawa H. Natural and induced T regulatory cells in cancer. Front Immunol. 2013;4:190

34. Marie JC, Letterio JJ, Gavin M, Rudensky AY. TGF-beta1 maintains suppressor function and Foxp3 expression in CD4 ${ }^{+} \mathrm{CD} 25^{+}$ regulatory T cells. J Exp Med. 2005;201(7):1061-1067.

35. Ghiringhelli F, et al. Tumor cells convert immature myeloid dendritic cells into TGF-beta-secreting cells inducing CD4 ${ }^{+} \mathrm{CD} 25^{+}$ regulatory T cell proliferation. J Exp Med. 2005;202(7):919-929.

36. Massagué J. TGF $\beta$ in Cancer. Cell. 2008;134(2):215-230.

37. Flavell RA, Sanjabi S, Wrzesinski SH, Licona-Limón P. The polarization of immune cells in the tumour environment by TGFbeta. Nat Rev Immunol. 2010;10(8):554-567.

38. Rubinson DA, et al. A lentivirus-based system to functionally silence genes in primary mammalian cells, stem cells and transgenic mice by RNA interference. Nat Genet. 2003;33(3):401-406.

39. Bergot AS, Durgeau A, Levacher B, Colombo BM, Cohen JL, Klatzmann D. Antigen quality determines the efficiency of antitumor immune responses generated in the absence of regulatory T cells. Cancer Gene Ther. 2010;17(9):645-654.

40. Gene Ontology Consortium. Creating the gene ontology resource: design and implementation. Genome Res. 2001;11(8):14251433.

41. Shannon P, et al. Cytoscape: a software environment for integrated models of biomolecular interaction networks. Genome Res. 2003;13(11):2498-2504. 
42. Merico D, Isserlin R, Stueker O, Emili A, Bader GD. Enrichment map: a network-based method for gene-set enrichment visualization and interpretation. PLoS ONE. 2010;5(11):e13984

43. Pham HP, Dérian N, Chaara W, Bellier B, Klatzmann D, Six A. A novel strategy for molecular signature discovery based on independent component analysis. Int J Data Min Bioinform. 2014;9(3):277-304.

44. Fisson S, et al. Continuous activation of autoreactive $\mathrm{CD} 4^{+} \mathrm{CD} 25^{+}$regulatory T cells in the steady state. J Exp Med. 2003;198(5):737-746.

45. Tanaka H, et al. Transforming growth factor $\beta$ signaling inhibitor, SB-431542, induces maturation of dendritic cells and enhances anti-tumor activity. Oncol Rep. 2010;24(6):1637-1643

46. Waghabi MC, et al. Pharmacological inhibition of transforming growth factor beta signaling decreases infection and prevents heart damage in acute Chagas' disease. Antimicrob Agents Chemother. 2009;53(11):4694-4701.

47. Bhaskaran N, Quigley C, Weinberg A, Huang A, Popkin D, Pandiyan P. Transforming growth factor- $\beta 1$ sustains the survival of Foxp3(+) regulatory cells during late phase of oropharyngeal candidiasis infection [published online ahead of print November 4, 2015]. Mucosal Immunol. doi: 10.1038/mi.2015.115.

48. Somasundaram R, Herlyn M. Nivolumab in combination with ipilimumab for the treatment of melanoma. Expert Rev Anticancer Ther. 2015;15(10):1135-1141.

49. Vanneman M, Dranoff G. Combining immunotherapy and targeted therapies in cancer treatment. Nat Rev Cancer. 2012;12(4):237-251

50. Chouaib S, Messai Y, Couve S, Escudier B, Hasmim M, Noman MZ. Hypoxia promotes tumor growth in linking angiogenesis to immune escape. Front Immunol. 2012;3:21

51. Hodi FS, et al. Bevacizumab plus ipilimumab in patients with metastatic melanoma. Cancer Immunol Res. 2014;2(7):632-642

52. Marzo AL, et al. Tumor antigens are constitutively presented in the draining lymph nodes. J Immunol. 1999;162(10):5838-5845.

53. Desjardins D, et al. Recombinant retrovirus-like particle forming DNA vaccines in prime-boost immunization and their use for hepatitis C virus vaccine development. J Gene Med. 2009;11(4):313-325.

54. Naldini L, et al. In vivo gene delivery and stable transduction of nondividing cells by a lentiviral vector. Science. 1996;272(5259):263-267.

55. Li H, et al. Vaccination with B16 tumor cell lysate plus recombinant Mycobacterium tuberculosis Hsp70 induces antimelanoma effect in mice. Cancer Biother Radiopharm. 2010;25(2):185-191.

56. Darrasse-Jèze G, Darasse-Jèze G, Klatzmann D, Charlotte F, Salomon BL, Cohen JL. CD4 $4^{+}$D $25^{+}$regulatory/suppressor T cells prevent allogeneic fetus rejection in mice. Immunol Lett. 2006;102(1):106-109.

57. Chaput $\mathrm{N}$, et al. Regulatory $\mathrm{T}$ cells prevent CD8 T cell maturation by inhibiting CD 4 Th cells at tumor sites. J Immunol. 2007;179(8):4969-4978.

58. Subramanian A, et al. Gene set enrichment analysis: a knowledge-based approach for interpreting genome-wide expression profiles. Proc Natl Acad Sci U S A. 2005;102(43):15545-15550. 